\title{
Updated evaluation of $\varepsilon_{K}$ in the standard model with lattice QCD inputs
}

\author{
Jon A. Bailey, ${ }^{1}$ Sunkyu Lee, ${ }^{1}$ Weonjong Lee,,${ }^{1,}$ Jaehoon Leem, ${ }^{2}$ and Sungwoo Park ${ }^{3}$ \\ (SWME Collaboration) \\ ${ }^{1}$ Lattice Gauge Theory Research Center, FPRD, and CTP, Department of Physics and Astronomy,
Seoul National University, Seoul 08826, South Korea
${ }^{2}$ School of Physics, Korea Institute for Advanced Study (KIAS), Seoul 02455, South Korea
${ }^{3}$ Los Alamos National Laboratory, Theoretical Division T-2, Los Alamos, New Mexico 87545, USA
}

(Received 4 September 2018; published 12 November 2018)

\begin{abstract}
We report a strong tension in $\varepsilon_{K}$ at the $4 \sigma$ level between the experimental value and the theoretical value calculated directly from the standard model using lattice QCD inputs such as $\hat{B}_{K},\left|V_{c b}\right|,\left|V_{u s}\right|, \xi_{0}, \xi_{2}, \xi_{\mathrm{LD}}$, $F_{K}$, and $m_{c}$. The standard model with lattice QCD inputs describes only $70 \%$ of the experimental value of $\varepsilon_{K}$, and does not explain its remaining $30 \%$. We also find that this tension disappears when we use the inclusive value of $\left|V_{c b}\right|$ (results of the heavy quark expansion based on QCD sum rules) to determine $\varepsilon_{K}$. This tension is highly correlated with the present discrepancy between the exclusive and inclusive values of $\left|V_{c b}\right|$. In order to resolve, in part, the issue with $\left|V_{c b}\right|$, it would be highly desirable to have a comprehensive reanalysis over the entire set of experimental data on the $\bar{B} \rightarrow D^{*} \ell \bar{\nu}$ decays using an alternative parametrization of the form factors, such as the Boyd, Grinstein, and Lebed parametrization, and a comparison with results of the Caprini, Lellouch, and Neubert method.
\end{abstract}

DOI: 10.1103/PhysRevD.98.094505

\section{INTRODUCTION}

$C P$ violation serves as a natural place to search for new physics [1,2]. The $C P$ violation in the neutral kaon system is, in particular, attractive to us, because the experimental results are already extremely precise [3], and lattice QCD allows us to perform a high precision calculation in kaon physics [4]. In this paper, we focus on the indirect $C P$ violation parameter $\varepsilon_{K}$, which we want to determine using lattice QCD inputs.

Indirect $C P$ violation in the neutral kaon system is parametrized by $\varepsilon_{K}$,

$$
\varepsilon_{K} \equiv \frac{\mathcal{A}\left(K_{L} \rightarrow \pi \pi(I=0)\right)}{\mathcal{A}\left(K_{S} \rightarrow \pi \pi(I=0)\right)}
$$

where $K_{L}$ and $K_{S}$ are the neutral kaon states in nature, and $I=0$ represents the isospin of the final two-pion state. In experiment [3],

\footnotetext{
*wlee@snu.ac.kr
}

Published by the American Physical Society under the terms of the Creative Commons Attribution 4.0 International license. Further distribution of this work must maintain attribution to the author(s) and the published article's title, journal citation, and DOI. Funded by SCOAP.

$$
\begin{aligned}
\varepsilon_{K} & =(2.228 \pm 0.011) \times 10^{-3} \times e^{i \phi_{\varepsilon}}, \\
\phi_{\varepsilon} & =43.52 \pm 0.05^{\circ}
\end{aligned}
$$

Here, the $\varepsilon_{K}$ value represents an $\approx 0.2 \%$ impurity of the $C P$ even eigenstate in the $K_{L}$ state, which contains $\approx 99.8 \%$ of the $C P$ odd eigenstate.

The standard model (SM) describes the $C P$ violation using a single phase in the CKM matrix elements. Hence, if there exists another phase coming from new physics, $\varepsilon_{K}$ is a natural place to find it, since $\varepsilon_{K}$ is highly sensitive to it. Therefore, it has been one of the top priorities in lattice QCD to calculate $\varepsilon_{K}$ to the highest possible precision [4].

In order to evaluate $\varepsilon_{K}$ directly from the SM, we need to know 18 input parameters [5]. Out of them, we can, in principle, obtain seven parameters from lattice QCD: $\hat{B}_{K},\left|V_{c b}\right|, \xi_{0}, \xi_{2}, \xi_{\mathrm{LD}},\left|V_{u s}\right|, m_{c}\left(m_{c}\right)$, and $F_{K}{ }^{1}$ During the last decade, lattice QCD has made such remarkable progress in calculating $\hat{B}_{K}$ that its error is only $1.3 \%$ at present [6]. At present, the largest error in theoretical calculation of $\varepsilon_{K}$ comes from $\left|V_{c b}\right|$ [5,7-9].

\footnotetext{
${ }^{1}$ In this number count, $\xi_{0}$ and $\xi_{2}$ are redundant. We need to know only $\xi_{0}$, but it is possible to obtain $\xi_{0}$ from $\xi_{2}$ using $\varepsilon^{\prime} / \varepsilon$. For more details, refer to Ref. [5].
} 
Here, we report the final results to draw your attention to the key issues. Evaluating $\varepsilon_{K}$ directly from the SM with lattice QCD inputs, we find that it has $4.2 \sigma-3.9 \sigma$ tension with the experimental result when we use exclusive ${ }^{2} .\left|V_{c b}\right|$ We also find that this tension disappears with inclusive $\left|V_{c b}\right|$. Hence, it is clear that the key issue is the input value of $\left|V_{c b}\right|$; the $4 \sigma$ tension in $\varepsilon_{K}$ is highly correlated with the $3 \sigma$ tension between exclusive and inclusive $\left|V_{c b}\right|$ [5].

At present, there are two independent methods to determine $\left|V_{c b}\right|$ : one is the exclusive method and the other is the inclusive method. In the exclusive method, the experimentalists use the exclusive decays $\bar{B} \rightarrow$ $D^{(*)} \ell \bar{\nu}$ to determine $\left|V_{c b}\right| \mathcal{F}(1)$, and then combine them with lattice QCD results for the form factor $\mathcal{F}(w)$ to determine $\left|V_{c b}\right|$ [10]. In the final analysis, they also include results for $\left|V_{c b}\right| /\left|V_{u b}\right|$ obtained by combining the $\mathrm{LHCb}$ results for the ratio of the branching fractions between the $\Lambda_{b} \rightarrow \Lambda_{c} \ell \bar{\nu}$ and $\Lambda_{b} \rightarrow p \ell \bar{\nu}$ decays with lattice QCD form factors [10]. In the inclusive method, one uses the heavy quark expansion as the theoretical framework to perform the data analysis on $\bar{B} \rightarrow X_{c} \ell \bar{\nu}$ decay processes [10]. The current status of $\left|V_{c b}\right|$ is, in units of $1.0 \times 10^{-3}$,

$$
\text { exclusive }\left|V_{c b}\right|=39.13 \pm 0.59 \text { from Ref. [10] }
$$

$$
\text { inclusive }\left|V_{c b}\right|=41.98 \pm 0.45 \text { from Ref. [10], }
$$

where the result in Eq. (4) is obtained in the $1 \mathrm{~S}$ scheme. The difference between (3) and (4) is 3.8 $\sigma$. This gap between exclusive and inclusive $\left|V_{c b}\right|$ has not been resolved yet. However, a number of interesting ideas have been proposed in order to resolve this issue $[11,12]$. We review them in Sec. III C 1 and Appendix A when we discuss $\left|V_{c b}\right|$.

The main goal of this paper is to present the most up-todate results for $\varepsilon_{K}$ obtained directly from the SM by using lattice QCD and experimental inputs. In Sec. II, we review the master formula for $\varepsilon_{K}$ and describe each term in detail, including the physical meaning. In Sec. III, we explain how to obtain the 18 input parameters one by one. In the case of $\left|V_{c b}\right|$, caveats in various methods for the form factor parametrization are addressed in some detail. In Sec. IV, we present results for $\varepsilon_{K}$ obtained using various combinations of input parameters. In Sec. V, we conclude.

\footnotetext{
${ }^{2}$ Here, the $4.2 \sigma$ tension is obtained with the estimate of RBC-UKQCD for $\xi_{\mathrm{LD}}$, while the $3.9 \sigma$ tension is obtained with the BGI estimate. For more details, refer to Sec. III E.
}

\section{REVIEW OF $\varepsilon_{K}$}

\section{A. Master formula: $\varepsilon_{K}$}

In the $\mathrm{SM}$, the direct $C P$ violation parameter $\varepsilon_{K}$ in the neutral kaon system can be reexpressed in terms of the well-known SM parameters as follows,

$$
\begin{aligned}
\varepsilon_{K}= & e^{i \theta} \sqrt{2} \sin \theta\left(C_{\varepsilon} X_{\mathrm{SD}} \hat{B}_{K}+\frac{\xi_{0}}{\sqrt{2}}+\xi_{\mathrm{LD}}\right) \\
& +\mathcal{O}\left(\omega \varepsilon^{\prime}\right)+\mathcal{O}\left(\xi_{0} \Gamma_{2} / \Gamma_{1}\right)
\end{aligned}
$$

This is the master formula, and its derivation is well explained in Ref. [5]. Here, we use the same notation and convention as in Ref. [5].

\section{B. Short distance contribution to $\varepsilon_{K}$}

In the master formula of Eq. (5), the dominant leadingorder effect $(\approx+107 \%)$ comes from the short distance (SD) contribution proportional to $\hat{B}_{K}$. Here, $C_{\varepsilon}$ is a dimensionless parameter defined as

$$
C_{\varepsilon} \equiv \frac{G_{F}^{2} F_{K}^{2} m_{K^{0}} M_{W}^{2}}{6 \sqrt{2} \pi^{2} \Delta M_{K}} \cong 3.63 \times 10^{4}
$$

Here, $X_{\mathrm{SD}}$ represents the short distance effect from the Inami-Lim functions [13],

$$
\begin{aligned}
X_{\mathrm{SD}} \equiv & \operatorname{Im} \lambda_{t}\left[\operatorname{Re} \lambda_{c} \eta_{c c} S_{0}\left(x_{c}\right)-\operatorname{Re} \lambda_{t} \eta_{t t} S_{0}\left(x_{t}\right)\right. \\
& \left.-\left(\operatorname{Re} \lambda_{c}-\operatorname{Re} \lambda_{t}\right) \eta_{c t} S_{0}\left(x_{c}, x_{t}\right)\right] \\
\cong & 6.24 \times 10^{-8}
\end{aligned}
$$

where $\lambda_{i}=V_{i s}^{*} V_{i d}$ is a product of the CKM matrix elements with $i=u, c, t$, and $\eta_{i j}$ with $i, j=c, t$ represent the QCD corrections of higher order in $\alpha_{s}$ [14]. There exists a potential issue with poor convergence of perturbation theory for $\eta_{c c}$ at the charm scale, which is discussed properly in Ref. [5]. Here, $S_{0}$ 's are Inami-Lim functions [13] defined as

$$
\begin{aligned}
S_{0}\left(x_{i}\right)= & x_{i}\left[\frac{1}{4}+\frac{9}{4\left(1-x_{i}\right)}-\frac{3}{2\left(1-x_{i}\right)^{2}}-\frac{3 x_{i}^{2} \ln x_{i}}{2\left(1-x_{i}\right)^{3}}\right], \\
S_{0}\left(x_{i}, x_{j}\right)= & \left\{\frac{x_{i} x_{j}}{x_{i}-x_{j}}\left[\frac{1}{4}+\frac{3}{2\left(1-x_{i}\right)}-\frac{3}{4\left(1-x_{i}\right)^{2}}\right] \ln x_{i}\right. \\
& +(i \leftrightarrow j)\}-\frac{3 x_{i} x_{j}}{4\left(1-x_{i}\right)\left(1-x_{j}\right)},
\end{aligned}
$$

where $i=c, t, x_{i}=m_{i}^{2} / M_{W}^{2}$, and $m_{i}=m_{i}\left(m_{i}\right)$ is the scale invariant (SI) $\overline{\mathrm{MS}}$ quark mass. In $X_{\mathrm{SD}}$ of Eq. (7), the $S_{0}\left(x_{t}\right)$ term from the top-top contribution in the box diagrams 
describes about $+72.4 \%$ of $X_{\mathrm{SD}}$, the $S_{0}\left(x_{c}, x_{t}\right)$ term from the top-charm contribution takes over about $+45.4 \%$ of $X_{\mathrm{SD}}$, and the $S_{0}\left(x_{c}\right)$ term from the charm-charm contribution depicts about $-17.8 \%$ of $X_{\mathrm{SD}}$.

Here, the kaon bag parameter $\hat{B}_{K}$ is defined as

$$
\begin{aligned}
\hat{B}_{K} & \equiv B_{K}(\mu) b(\mu) \cong 0.76, \\
B_{K}(\mu) & \equiv \frac{\left\langle\bar{K}^{0}\left|O_{L L}^{\Delta S=2}(\mu)\right| K^{0}\right\rangle}{\frac{8}{3}\left\langle\bar{K}^{0}\left|\bar{s} \gamma_{\mu} \gamma_{5} d\right| 0\right\rangle\left\langle 0\left|\bar{s} \gamma^{\mu} \gamma_{5} d\right| K^{0}\right\rangle} \\
& =\frac{\left\langle\bar{K}^{0}\left|O_{L L}^{\Delta S=2}(\mu)\right| K^{0}\right\rangle}{\frac{8}{3} F_{K}^{2} m_{K^{0}}^{2}}, \\
O_{L L}^{\Delta S=2}(\mu) & \equiv\left[\bar{s} \gamma_{\mu}\left(1-\gamma_{5}\right) d\right]\left[\bar{s} \gamma^{\mu}\left(1-\gamma_{5}\right) d\right],
\end{aligned}
$$

where $b(\mu)$ is the renormalization group running factor to make $\hat{B}_{K}$ invariant with respect to the renormalization scale and scheme,

$$
b(\mu)=\left[\alpha_{s}^{(3)}(\mu)\right]^{-2 / 9} K_{+}(\mu) .
$$

Here, details on $K_{+}(\mu)$ are given in Ref. [5].

\section{Long distance contribution to $\varepsilon_{K}$}

There are two kinds of long distance (LD) contributions on $\varepsilon_{K}$ : one is the absorptive LD effect from $\xi_{0}$ and the other is the dispersive LD effect from $\xi_{\mathrm{LD}}$. The absorptive LD effects are defined as

$$
\begin{aligned}
\tan \xi_{0} & \equiv \frac{\operatorname{Im} A_{0}}{\operatorname{Re} A_{0}}, \\
\tan \xi_{2} & \equiv \frac{\operatorname{Im} A_{2}}{\operatorname{Re} A_{2}} .
\end{aligned}
$$

They are related with each other through $\varepsilon^{\prime}$,

$$
\begin{aligned}
\varepsilon^{\prime} & \equiv e^{i\left(\delta_{2}-\delta_{0}\right)} \frac{i \omega}{\sqrt{2}}\left(\tan \xi_{2}-\tan \xi_{0}\right) \\
& =e^{i\left(\delta_{2}-\delta_{0}\right)} \frac{i \omega}{\sqrt{2}}\left(\xi_{2}-\xi_{0}\right)+\mathcal{O}\left(\xi_{i}^{3}\right) .
\end{aligned}
$$

The overall contribution of the $\xi_{0}$ term to $\varepsilon_{K}$ is about $-7 \%$.

The dispersive LD effect is defined as

$$
\xi_{\mathrm{LD}}=\frac{m_{\mathrm{LD}}^{\prime}}{\sqrt{2} \Delta M_{K}}
$$

where

$$
m_{\mathrm{LD}}^{\prime}=-\operatorname{Im}\left[\mathcal{P} \sum_{C} \frac{\left\langle\bar{K}^{0}\left|H_{\mathrm{w}}\right| C\right\rangle\left\langle C\left|H_{\mathrm{w}}\right| K^{0}\right\rangle}{m_{K^{0}}-E_{C}}\right],
$$

if the $C P T$ invariance is well respected. The overall contribution of the $\xi_{\mathrm{LD}}$ to $\varepsilon_{K}$ is about $\pm 2 \%$.

\section{Erratum}

There were two pure typos in Ref. [5]. One typo is found in Eq. (50) of Ref. [5]. The correct equations for $S_{0}\left(x_{i}\right)$ and $S_{0}\left(x_{i}, x_{j}\right)$ are given in Eq. (9) of this paper. The other typo is found in Eq. (62) of Ref. [5]. The correct equation for $\delta m_{\mathrm{LD}}^{\prime}$ is

$$
\delta m_{\mathrm{LD}}^{\prime}=-i \frac{1}{2} \mathcal{P} \sum_{C} \frac{\left|\left\langle K_{0}\left|H_{\mathrm{w}}\right| C\right\rangle\right|^{2}-\left|\left\langle\bar{K}^{0}\left|H_{\mathrm{w}}\right| C\right\rangle\right|^{2}}{m_{K^{0}}-E_{C}}=0,
$$

if the $C P T$ invariance is well respected. The $-i$ factor is missing in Ref. [5]. In our actual calculation of $\varepsilon_{K}$, we used the correct equations with no mistake, even though we introduced the above two typos in writing up the paper of Ref. [5].

\section{INPUT PARAMETERS}

We need to know values of 18 parameters defined in the SM in order to evaluate $\varepsilon_{K}$ directly from the SM. Out of the 18 parameters, we can obtain, in principle, seven parameters such as $\hat{B}_{K},\left|V_{c b}\right|, \xi_{0}, \xi_{2}, \xi_{\mathrm{LD}},\left|V_{u s}\right|, F_{K}$, and $m_{c}\left(m_{c}\right)$ directly from lattice QCD. Here, we describe how to obtain the 18 input parameters from the experiments and from lattice QCD results in detail.

\section{A. Wolfenstein parameters}

The CKMfitter [15] and UTfit [16] collaborations provide the Wolfenstein parameters [17] $(\lambda, \bar{\rho}, \bar{\eta})$ determined by the global unitarity triangle (UT) fit. The 2017 results are summarized in Table I. As pointed out in Ref. [5], the Wolfenstein parameters extracted by the global UT fit have unwanted correlation with $\varepsilon_{K}$, because $\varepsilon_{K}$ is used as an input to obtain them. Hence, in order to avoid this correlation, we take another set of the Wolfenstein parameters determined from the angle-only-fit (AOF) suggested in Ref. [18]. In the AOF, $\varepsilon_{K}, \hat{B}_{K}$, and $\left|V_{c b}\right|$ are not used as inputs to determine the UT apex $(\bar{\rho}, \bar{\eta})$. Then, we determine $\lambda$ from $\left|V_{u s}\right|$ which is obtained from the $K_{\ell 2}$ and $K_{\ell 3}$ decays using the lattice QCD results.

TABLE I. Wolfenstein parameters (WP). Both CKMfitter and UTfit groups use the global unitarity triangle fit. Here, AOF represents the angle only fit.

\begin{tabular}{lllllll}
\hline \hline WP & \multicolumn{2}{c}{ CKMfitter } & UTfit & \multicolumn{2}{c}{ AOF } \\
\hline$\lambda$ & $0.22509(29)$ & {$[15]$} & $0.22497(69)$ & {$[16]$} & $0.2248(6)$ & {$[3]$} \\
$\bar{\rho}$ & $0.1598(76)$ & {$[15]$} & $0.153(13)$ & {$[16]$} & $0.146(22)$ & {$[19]$} \\
$\bar{\eta}$ & $0.3499(63)$ & {$[15]$} & $0.343(11)$ & {$[16]$} & $0.333(16)$ & {$[19]$} \\
\hline \hline
\end{tabular}


TABLE II. $\quad \hat{B}_{K}$ in lattice QCD with $N_{f}=2+1$.

\begin{tabular}{lll}
\hline \hline Collaboration & Ref. & \multicolumn{1}{c}{$\hat{B}_{K}$} \\
\hline SWME 15 & {$[26]$} & $0.735(5)(36)$ \\
RBC/UKQCD 14 & {$[25]$} & $0.7499(24)(150)$ \\
Laiho 11 & {$[24]$} & $0.7628(38)(205)$ \\
BMW 11 & {$[23]$} & $0.7727(81)(84)$ \\
FLAG 17 & {$[6]$} & $0.7625(97)$ \\
\hline \hline
\end{tabular}

The Wolfenstein parameter $A$ is determined directly from $\left|V_{c b}\right|$, which is discussed later in Sec. IIIC. The Wolfenstein parameters from the AOF are summarized in Table I.

\section{B. $\hat{\boldsymbol{B}}_{K}$}

In the FLAG review [6], they present lattice QCD results for $\hat{B}_{K}$ with $N_{f}=2, N_{f}=2+1$, and $N_{f}=2+1+1$. Recent calculations of $\hat{B}_{K}$ in lattice QCD have been done with $N_{f}=2+1+1$ dynamical quarks [20]. We do not prefer it for two physical reasons.

(1) The master formula in Eq. (5) is derived by integrating out the heavy particles including the charm quark. Hence, it is complicated and inconvenient to use the results for $\hat{B}_{K}$ with dynamical charm quarks $[6,20]$.

(2) A proper and systematic procedure to incorporate the charm quark effect in the calculation of $\varepsilon_{K}$ is available in Refs. [21,22]. However, this new method is in the stage of exploratory study and has not reached the stage of precision measurement yet.

Similarly, we prefer using the results for $\hat{B}_{K}$ with $N_{f}=$ $2+1$ to those with $N_{f}=2$ because they are obtained by quenching the vacuum polarization contributions of the strange quark.

In Table II, we present the FLAG results for $\hat{B}_{K}$ with $N_{f}=2+1$. Here, they take a global average over the four data points from BMW 11 [23], Laiho 11 [24], RBC/UKQCD 14 [25], and SWME 15 [26]. The FLAG 17 in the table represents the final result for $\hat{B}_{K}$. Here, we use this for our evaluation of $\varepsilon_{K}$.

$$
\text { C. }\left|\boldsymbol{V}_{\boldsymbol{c} b}\right|
$$

In Table III, we summarize updated results for both exclusive $\left|V_{c b}\right|$ and inclusive $\left|V_{c b}\right|$. Recently HFLAV reported them in Ref. [10]. The results for exclusive $\left|V_{c b}\right|$ depend on the lattice QCD calculations of form factors of Refs. [27-29]. Here, when we obtain the in-combined results in Table III(b), we neglect the hidden correlation of the inclusive $\left|V_{c b}\right|$ between the kinetic scheme and the $1 \mathrm{~S}$ scheme, even though there must be some correlation because they share some experimental data with each other. Hence, we prefer using results of the $1 \mathrm{~S}$ scheme to the results of
TABLE III. Results for $\left|V_{c b}\right|$ in units of $1.0 \times 10^{-3}$. The incombined result is obtained by taking an uncorrelated weighted average of the two values in Table (b).

\begin{tabular}{lcc}
\hline \hline & (a) Exclusive $\left|V_{c b}\right|$ & \\
\hline Channel & Value & Ref. \\
\hline$B \rightarrow D^{*} \ell \bar{\nu}$ & $39.05(47)(58)$ & {$[10]^{\mathrm{a}}$} \\
$B \rightarrow D \ell \bar{\nu}$ & $39.18(94)(36)$ & {$[10]^{\mathrm{b}}$} \\
$\left|V_{u b}\right| /\left|V_{c b}\right|$ & $0.080(4)(4)$ & {$[10]^{\mathrm{c}}$} \\
Ex-combined & $39.13(59)$ & {$[10]$} \\
& (b) Inclusive $\left|V_{c b}\right|$ & \\
\hline Channel & Value & Ref. \\
\hline Kinetic scheme & $42.19(78)$ & {$[10]$} \\
$1 S$ scheme & $41.98(45)$ & {$[10]$} \\
In-combined & $42.03(39)$ & This paper \\
\hline \hline
\end{tabular}

${ }^{\mathrm{a}}$ In this analysis, they use the lattice QCD results for the semileptonic form factors in Ref. [27].

${ }^{\mathrm{b}}$ In this analysis, they use the lattice QCD results for the semileptonic form factors in Ref. [28].

'In this analysis, they use the lattice QCD results for the semileptonic form factors in Ref. [29].

in-combined here. We use the combined results (excombined) for the exclusive $\left|V_{c b}\right|$ and the results of the $1 \mathrm{~S}$ scheme for inclusive $\left|V_{c b}\right|$ when we evaluate $\varepsilon_{K}$.

In Fig. 1, we present results for $\left|V_{c b}\right|$ and $\left|V_{u b}\right|$. The big change is that, as of Lattice 2016, the result for exclusive

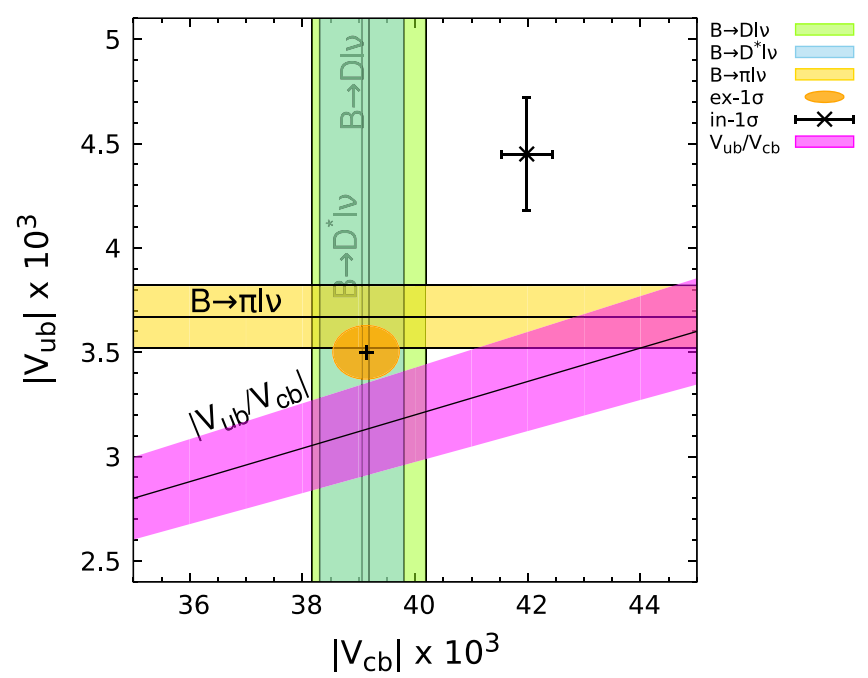

FIG. 1. $\left|V_{c b}\right|$ versus $\left|V_{u b}\right|$ in units of $1.0 \times 10^{-3}$. The light-blue band represents $\left|V_{c b}\right|$ determined from the $\bar{B} \rightarrow D^{*} \ell \bar{\nu}$ decay mode. The light-green band represents $\left|V_{c b}\right|$ determined from the $\bar{B} \rightarrow D \ell \bar{\nu}$ decay mode. The yellow band represents $\left|V_{u b}\right|$ determined from the $\bar{B} \rightarrow \pi \ell \bar{\nu}$ decay mode. The magenta band represents $\left|V_{u b} / V_{c b}\right|$ determined from the LHCb data of the $\Lambda_{b} \rightarrow \Lambda_{c} \ell \bar{\nu}$ and $\Lambda_{b} \rightarrow p \ell \bar{\nu}$ decay modes. The orange circle represents the combined results for exclusive $\left|V_{c b}\right|$ and $\left|V_{u b}\right|$ from the $B$ meson and $\Lambda_{b}$ decays within $1.0 \sigma$. The black cross $(\times)$ represents the inclusive $\left|V_{c b}\right|$ and $\left|V_{u b}\right|$ from the heavy quark expansion. The details are given in Table III. 
$\left|V_{c b}\right|$ from $\bar{B} \rightarrow D \ell \bar{\nu}$ was about one sigma away from that from $\bar{B} \rightarrow D^{*} \ell \bar{\nu}$ (refer to Refs. [8,9] for more details), but in 2017, they are on top of each other, as shown in Fig. 1. The 2017 results for $\bar{B} \rightarrow D^{*} \ell \bar{\nu}$ are not visibly different, but those for $\bar{B} \rightarrow D \ell \bar{\nu}$ shift downward by about $1 \sigma$. The difference is due to several factors acting in concert: The 2017 results of HFLAV include all results from the $B$ factories, BABAR and BELLE, as well as the older results from CLEO and the LEP experiments ALEPH, OPAL, and DELPHI. Before the results are averaged, they are rescaled by HFLAV to updated values of the inputs, and the averages include the effects of correlations.

To obtain the 2017 results for exclusive $\left|V_{c b}\right|$ (and $\left|V_{u b}\right|$ ), HFLAV performed a combined fit to all results for the decays $\bar{B} \rightarrow D^{(*)} \ell \bar{\nu}, B \rightarrow \pi \ell \nu$, and the ratio of the branching fractions for $\Lambda_{b} \rightarrow p \ell \bar{\nu}$ and $\Lambda_{b} \rightarrow \Lambda_{c} \ell \bar{\nu}$. With lattice QCD results for the form factors, the $B \rightarrow \pi \ell \nu$ decay yields $\left|V_{u b}\right|$, while the ratio of the branching fractions of the $\Lambda_{b}$ decays yields $\left|V_{u b}\right| /\left|V_{c b}\right|$. Due to the addition of more data to the HFLAV analysis, the results for $\left|V_{u b}\right| /\left|V_{c b}\right|$ shift downward by about $\frac{3}{4} \sigma$ in 2017 , while those for $\left|V_{u b}\right|$ shift downward by about $0.1 \sigma$. For more details, refer to Ref. [10].

\section{Caveats on CLN and BGL}

In order to extract a value of $\left|V_{c b}\right| \mathcal{F}(1)[\mathcal{F}(w)$ is a form factor at a recoil point $w]$ from the experiment of $\bar{B} \rightarrow$ $D^{(*)} \ell \bar{\nu}$ we need to know the functional form of the form factors as a function of $w$. There have been two kinds of parametrization methods developed to do this job: one is a heavy quark effective theory (HQET)-dependent method, and the other is an HQET-independent method. The former is a method of Caprini, Lellouch, and Neubert (CLN) in Ref. [30] and its sibling paper [31], and the latter is a method of Boyd, Grinstein, and Lebed (BGL) in Ref. [32] and its sibling papers [33-35]. ${ }^{3}$ Both of them have been developed on top of the building blocks designed for $K_{\ell 3}$ decays in Refs. [37-39].

Recently, in Refs. $[11,12,40]$, they claim that the gap between the inclusive $\left|V_{c b}\right|$ and exclusive $\left|V_{c b}\right|$ might be explained in part by the observation of both groups that CLN consistently underestimates the value of exclusive $\left|V_{c b}\right|$ compared with that of BGL. In this claim, they refer to the numbers of HFLAV in Ref. [10] which are obtained using the CLN method. Certainly, this claim is interesting enough to deserve our full and careful investigation of it.

Let us first describe the key points of the claim in Refs. $[11,12]$. In the CLN parametrization, they introduce the form factor $h_{A_{1}}(w)$, and the ratios of $R_{1}(w)$ and $R_{2}(w)$ to describe the form factors for $\bar{B} \rightarrow D^{*} \ell \bar{\nu}$ decays. Their definition [Eq. (A4)] and detailed explanation are given in

\footnotetext{
${ }^{3}$ There exists a variant of the BGL method which is often referred to as the "BCL method" [36].
}

Appendix A. Let us directly address the problematic part in CLN. CLN is constructed based on HQET and its perturbative application to the slope and curvature of $h_{A_{1}}(w), R_{1}(w)$, and $R_{2}(w)$. CLN was originally designed to have its error in the level of about $2 \%$ precision [30]. At present, the trouble is that the experimental precision goes below the $2 \%$ level. The lattice QCD results have precision better than that of the $2 \%$ level. The typical size of errors from the slope and curvature in $R_{i}(w)$ obtained using the perturbation theory in HQET is about $10 \%$ which has a potential to cause $1 \%-2 \%$ errors in $\left|V_{c b}\right|$. They $([11,12])$ observed that the CLN method consistently underestimates exclusive $\left|V_{c b}\right|$ compared with that of the BGL method which is model independent by construction. Details on BGL are summarized in Appendix A. To support their claim, they used preliminary unfolded data of BELLE in Ref. [41]. In their conclusion, they recommended comprehensive reanalysis of old experimental data used in Ref. [10] using the BGL method as well as some suggestions to the lattice QCD community.

In Ref. [42], they incorporate all the $\mathcal{O}\left(\Lambda_{\mathrm{QCD}} / m_{c, b}\right)$ and $\mathcal{O}\left(\alpha_{s}\right)$ contributions in the HQET framework into their analysis for $\left|V_{c b}\right|$ based on the CLN method. They find that their results for $\left|V_{c b}\right|$ with improved precision agree with those of HFLAV [10]. In Ref. [43], they use the same kind of CLN method as in Ref. [42] and its variations as well as the BGL method to determine $\left|V_{c b}\right|$ and semileptonic form factors. In this study they find that the slope of the form factor ratio $R_{1}(w)$ at zero recoil obtained using the BGL method has potentially large deviation from heavy quark symmetry, and, in addition, has significant tension with the preliminary lattice QCD results of FNAL/MILC [44] and JLQCD $[45,46]$. They point out that the tensions between the exclusive and inclusive determinations of $\left|V_{c b}\right|$ are far away from being considered resolved at present. In Ref. [40], however, they claim that the conclusions previously reached in Ref. [11] are not changed by taking into account heavy quark symmetry. The extraction of $\left|V_{c b}\right|$ using the CLN and BGL parametrizations and preliminary BELLE data has been further investigated in Ref. [47].

The claim in Refs. [11,12] is interesting, but far away from conclusive or decisive in that they used only a preliminary subset of the BELLE data, and the BGL results for the $R_{1}(w)$ slope have significant violation of heavy quark symmetry and are disfavored by preliminary lattice QCD results [43]. This issue might well be resolved one way or the other, once the next round of comprehensive reanalysis by HFLAV on the old experimental data used in Ref. [10] becomes available. Lattice QCD calculation of semileptonic form factors for the $\bar{B} \rightarrow D^{(*)} \ell \bar{\nu}$ decays at nonzero recoil will be helpful [45]. Hence, please stay tuned for this coming update.

\section{D. $\xi_{0}$}

The absorptive part of long distance effects in $\varepsilon_{K}$ is parametrized into $\xi_{0}$. We can express $\varepsilon^{\prime} / \varepsilon$ in terms of $\xi_{0}$ and $\xi_{2}$ as follows, 
TABLE IV. Input parameter $\xi_{0}$.

\begin{tabular}{llcc}
\hline \hline Parameter & Method & Value & Ref. \\
\hline$\xi_{0}$ & Indirect & $-1.63(19) \times 10^{-4}$ & {$[48]$} \\
$\xi_{0}$ & Direct & $-0.57(49) \times 10^{-4}$ & {$[49]$} \\
\hline \hline
\end{tabular}

$$
\begin{gathered}
\xi_{0} \equiv \arctan \left(\frac{\operatorname{Im} A_{0}}{\operatorname{Re} A_{0}}\right)=\frac{\operatorname{Im} A_{0}}{\operatorname{Re} A_{0}}+\mathcal{O}\left(\xi_{0}^{3}\right) \\
\xi_{2} \equiv \arctan \left(\frac{\operatorname{Im} A_{2}}{\operatorname{Re} A_{2}}\right)=\frac{\operatorname{Im} A_{2}}{\operatorname{Re} A_{2}}+\mathcal{O}\left(\xi_{2}^{3}\right) \\
\operatorname{Re}\left(\frac{\varepsilon^{\prime}}{\varepsilon}\right)=\frac{\omega}{\sqrt{2}\left|\varepsilon_{K}\right|}\left(\xi_{2}-\xi_{0}\right) .
\end{gathered}
$$

There are two independent methods to determine $\xi_{0}$ in lattice QCD: one is the indirect method and the other is the direct method. In the indirect method, we determine $\xi_{0}$ using Eq. (22) with lattice QCD input $\xi_{2}$ and with experimental results for $\varepsilon^{\prime} / \varepsilon, \varepsilon_{K}$, and $\omega$. In the direct method, we can determine $\xi_{0}$ directly using lattice QCD results for $\operatorname{Im} A_{0}$ combined with experimental results for $\operatorname{Re} A_{0}$.

Recently, RBC-UKQCD reported results for $\xi_{2}$ in Ref. [48]. Using the indirect method, we can obtain the result for $\xi_{0}$ as in Table IV. Recently, RBC-UKQCD also reported results for $\operatorname{Im} A_{0}$ in Ref. [49]. Using the experimental value of $\operatorname{Re} A_{0}$, we can obtain $\xi_{0}$ directly from $\operatorname{Im} A_{0}$, which is summarized in Table IV.

In Ref. [49] RBC-UKQCD also reported the S-wave $\pi-\pi$ scattering phase shift for the $I=0$ channel: $\delta_{0}=23.8(49)(12)$. This value is $3.0 \sigma$ lower than the conventional results for $\delta_{0}$ in Refs. [50] (KPY-2011) and [51,52] (CGL-2001), and [53]. The values for $\delta_{0}$ are summarized in Table V.

In Fig. 2, we show the experimental results for $\delta_{0}$ with the fitting results of KPY-2011. They (KPY-2011) used a singly subtracted Roy-like equation to do the interpolation around $\sqrt{s}=m_{K}$ (the physical kaon mass). Their fitting to the experimental data works well from the threshold to $\sqrt{s} \cong 800 \mathrm{MeV}$. In this range they use the singly subtracted Roy-like equation to do the fitting.

In Fig. 3, we show the fitting results of both KPY-2011 and CGL-2001 as well as the results of RBC-UKQCD. There is essentially no difference between KPY-2011 and CGL-2001 in the region near $\sqrt{s}=m_{K}$. As one can see in

TABLE V. Results for $\delta_{0}$

\begin{tabular}{llc}
\hline \hline Collaboration & \multicolumn{1}{c}{$\delta_{0}$} & Ref. \\
\hline RBC-UKQCD-2016 & $23.8(49)(12)^{\circ}$ & {$[49]$} \\
KPY-2011 & $39.1(6)^{\circ}$ & {$[50]$} \\
CGL-2001 & $39.2(15)^{\circ}$ & {$[51,52]$} \\
\hline \hline
\end{tabular}

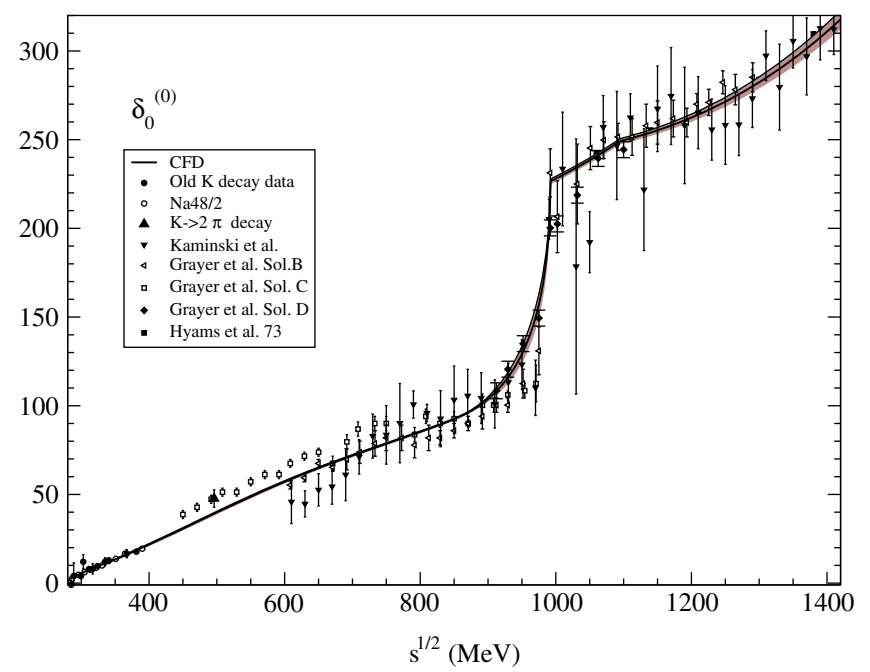

FIG. 2. Experimental results for $\delta_{0}$. We borrow this plot from Ref. [50].

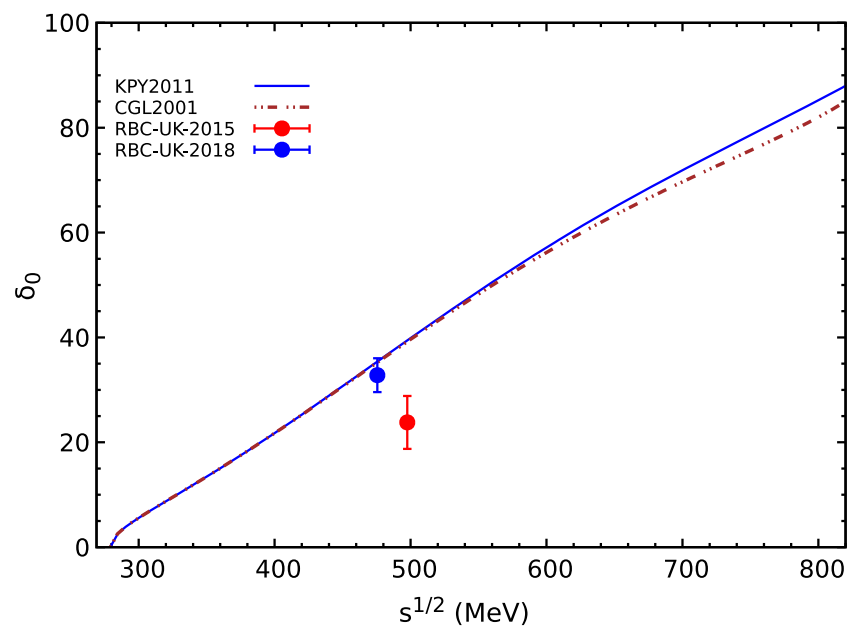

(a) $\delta_{0}$

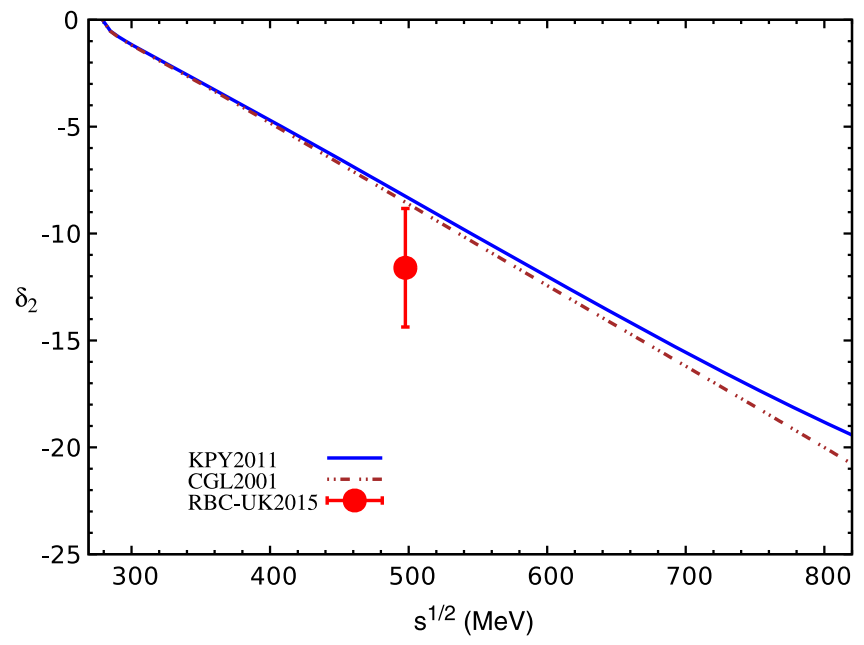

(b) $\delta_{2}$

FIG. 3. S-wave $\pi-\pi$ scattering phase shifts $\delta_{I}$ for (a) $I=0$ and (b) $I=2$ channels. 
Fig. 3(a), we observe the $3.0 \sigma$ tension for $\delta_{0}$ between RBCUKQCD and KPY-2011. In the case of $\delta_{2}$, there is no difference between RBC-UKQCD and KPY-2011 within statistical uncertainty as one can see in Fig. 3(b). Taking into account all the aspects, we conclude that the direct calculation of $\operatorname{Im} A_{0}$ and $\xi_{0}$ by RBC-UKQCD in Ref. [49] might have unresolved issues. Indeed, preliminary results presented by RBC-UKQCD in Lattice 2018 suggest that this discrepancy might disappear with improved analysis [54].

Therefore, we prefer the indirect method to the direct method for the following two reasons. The first reason is that the lattice QCD calculation of $\operatorname{Im} A_{0}$ is much noisier than that of $\operatorname{Im} A_{2}$ thanks to many disconnected diagrams. The second reason is that the S-wave phase shift $\delta_{0}$ of the $\pi-\pi$ scattering in Ref. [49] is lower by $3.0 \sigma$ than the conventional determination of $\delta_{0}$ in Refs. [50,51,53], which indicates that there might be some issues unresolved at present. In Table IV, we present results of $\xi_{0}$ determined using both indirect and direct methods. Here, we use the value of $\xi_{0}$ obtained using the indirect method.

One remaining caveat is that the $\xi_{0}$ and $\xi_{2}$ in Ref. [48] are calculated in the isospin symmetric limit. The isospin breaking effects on $\varepsilon^{\prime} / \varepsilon$ are studied in Refs. [55,56]. These studies conclude that the isospin violation correction in the $C P$ violation correction for $\varepsilon^{\prime}$ is below $15 \%$ within the uncertainties of large $N_{c}$ estimates for the low energy constants. Since $\xi_{0}$ has an effect of about $-7 \%$ on $\varepsilon_{K}$, the isospin violation effect maximum, $15 \%$ of $\xi_{0}$ amounts to $\pm 1 \%$ correction for $\varepsilon_{K}$. Here, we neglect this effect completely without loss of generality in our conclusion.

\section{E. $\boldsymbol{\xi}_{\mathrm{LD}}$}

The LD effects on $\varepsilon_{K}$ are explained in Sec. II C. Hence, here we summarize the recent progress in calculating the LD effects in lattice QCD.

Lattice QCD tools to calculate the dispersive LD effect, $\xi_{\text {LD }}$ are well established in Refs. [21,22,57]. In addition, recently, there have been a number of attempts to calculate $\xi_{\mathrm{LD}}$ on the lattice [58,59]. In these attempts, RBC-UKQCD used pion mass of $329 \mathrm{MeV}$ and kaon mass of $591 \mathrm{MeV}$. Hence, the energy of the two-pion state and three pion states is heavier than the kaon mass. Therefore, the sign of the denominator in Eq. (18) is opposite to that of the physical contribution in which the two and three pion state energy is lighter than the kaon mass. Therefore, this attempt in Refs. [58,59] belongs to the category of exploratory study rather than to that of precision measurement.

The net contribution of $\xi_{\mathrm{LD}}$ to $\varepsilon_{K}$ in Eqs. (17) and (18) turns out to be of the same order of magnitude as $\xi_{0}$ using chiral perturbation theory [60]. The authors claim that

$$
\xi_{\mathrm{LD}}=-0.4(3) \times \frac{\xi_{0}}{\sqrt{2}},
$$

where we use the indirect results of $\xi_{0}$ given in Table IV, including its error. Here, we call this method the BGI estimate for $\xi_{\mathrm{LD}}$. This also indicates that $\xi_{\mathrm{LD}}$ is at most a $4 \%$ correction to $\varepsilon_{K}$. This claim is highly consistent with the estimate of about $2 \%$ in Refs. [21,61],

$$
\xi_{\mathrm{LD}}=(0 \pm 1.6) \% .
$$

Here, we call this method the RBC-UKQCD estimate for $\xi_{\mathrm{LD}}$.

In this paper, we use both of the above estimates of $\xi_{\mathrm{LD}}$ with the BGI and RBC-UKQCD methods to determine $\varepsilon_{K}$.

\section{F. Top quark mass}

The pole mass of top quarks coming from Ref. [3] is

$$
M_{t}=173.5 \pm 1.1 \mathrm{GeV} .
$$

The pole and $\overline{\mathrm{MS}}$ masses are related as follows,

$$
\frac{m_{t}(\mu)}{M_{t}}=z(\mu)=\frac{Z_{\mathrm{OS}}}{Z_{\overline{\mathrm{MS}}}}
$$

where $m_{t}(\mu)$ is the $\overline{\mathrm{MS}}$ mass renormalized at scale $\mu$. Here, $Z_{\mathrm{OS}}$ is the renormalization factor in the on-shell scheme, and $Z_{\overline{\mathrm{MS}}}$ is the renormalization factor in the $\overline{\mathrm{MS}}$ scheme. The top scale-invariant quark mass $\mu_{t}$ is the $\overline{\mathrm{MS}}$ mass $m_{t}(\mu)$ with the scale $\mu$ set equal to the scale-invariant mass,

$$
\mu_{t}=m_{t}\left(\mu_{t}\right)=163.65 \pm 1.05 \pm 0.17 \mathrm{GeV}
$$

where we use the four-loop perturbation formula for $z\left(\mu_{t}\right)$. Details on the four-loop conversion formula are described in Appendix B. In Eq. (27), the first error comes from the error of the top pole mass, and the second error represents the uncertainty due to truncation of higher loops in the conversion formula which is estimated as the difference in $\mu_{t}$ between the three-loop and four-loop formulas. We have neglected the renormalon ambiguity and corrections due to the three-loop fermion mass such as $m_{b}$ (bottom quark mass) and $m_{c}$ (charm quark mass).

\section{G. Other input parameters}

For the higher order QCD corrections $\eta_{c c}, \eta_{c t}$, and $\eta_{t t}$, we use the same values as in Ref. [5]. They are summarized in Table VI.

TABLE VI. Higher order QCD corrections: $\eta_{c c}, \eta_{t t}$, and $\eta_{c t}$.

\begin{tabular}{llc}
\hline \hline Input & \multicolumn{1}{c}{ Value } & Ref. \\
\hline$\eta_{c c}$ & $1.72(27)$ & {$[5]$} \\
$\eta_{t t}$ & $0.5765(65)$ & {$[62]$} \\
$\eta_{c t}$ & $0.496(47)$ & {$[63]$} \\
\hline \hline
\end{tabular}


TABLE VII. Other input parameters.

\begin{tabular}{lcc}
\hline \hline Input & Value & Ref. \\
\hline$G_{F}$ & $1.1663787(6) \times 10^{-5} \mathrm{GeV}^{-2}$ & {$[3]$} \\
$M_{W}$ & $80.385(15) \mathrm{GeV}$ & {$[3]$} \\
$m_{c}\left(m_{c}\right)$ & $1.2733(76) \mathrm{GeV}$ & {$[64]$} \\
$\theta$ & $43.52(5)^{\circ}$ & {$[3]$} \\
$m_{K^{0}}$ & {$[3]$} \\
$\Delta M_{K}$ & $497.611(13) \mathrm{MeV}$ & {$[3]$} \\
$F_{K}$ & $15.484(6) \times 10^{-12} \mathrm{MeV}$ & {$[3]$} \\
\hline \hline
\end{tabular}

Other input parameters are summarized in Table VII. They are the same as Ref. [5] except for charm quark mass $m_{c}\left(m_{c}\right)$, the kaon mass $m_{K^{0}}$, and the kaon decay constant $F_{K}$. For the charm quark mass, we use the HPQCD results of $m_{c}\left(m_{c}\right)$ reported in Ref. [64]. For the kaon mass, we use the updated results of Particle Data Group (PDG) in Ref. [3]. For the kaon decay constant, we use the updated results of PDG reported in Ref. [3], which are obtained from the FLAG data [6].

\section{RESULTS}

\section{A. RBC-UKQCD estimate for $\boldsymbol{\xi}_{\mathrm{LD}}$}

In Fig. 4, we present results for $\varepsilon_{K}$ calculated directly from the standard model with the lattice QCD inputs described in Sec. III. In Fig. 4(a), the blue curve which encircles the histogram represents the theoretical evaluation of $\varepsilon_{K}$ using the FLAG-2017 $\hat{B}_{K}$, AOF for Wolfenstein parameters, and exclusive $\left|V_{c b}\right|$ which corresponds to excombined in Table III(a), and the RBC-UKQCD estimate for $\xi_{\mathrm{LD}}$. The red curve in Fig. 4 represents the experimental result for $\varepsilon_{K}$. In Fig. 4(b), the blue curve represents the same
TABLE VIII. $\left|\varepsilon_{K}\right|$ values in units of $1.0 \times 10^{-3}$. The superscript SM represents the standard model. The subscript excl (incl) represents exclusive (inclusive) $\left|V_{c b}\right|$. The superscript Exp represents the experimental result. We use the same input parameters as in Fig. 4.

\begin{tabular}{llc}
\hline \hline Parameter & \multicolumn{1}{c}{ Method } & Value \\
\hline$\left|\varepsilon_{K}\right| \begin{array}{cc}\text { Sec } \\
\text { lecl }\end{array}$ & Exclusive $\left|V_{c b}\right|$ & $1.570 \pm 0.156$ \\
$\left|\varepsilon_{K}\right| \begin{array}{l}\text { SMcl } \\
\text { incl }\end{array}$ & Inclusive $\left|V_{c b}\right|$ & $2.035 \pm 0.178$ \\
$\left|\varepsilon_{K}\right|^{\text {Exp }}$ & Experiment & $2.228 \pm 0.011$ \\
\hline
\end{tabular}

as in Fig. 4(a) except for using the inclusive $\left|V_{c b}\right|$ which corresponds to $1 \mathrm{~S}$ scheme in Table III(b).

The updated results for $\left|\varepsilon_{K}\right|$ are, in units of $1.0 \times 10^{-3}$, presented in Table VIII. From Table VIII, we observe that the theoretical evaluation of $\left|\varepsilon_{K}\right|$ with lattice QCD inputs (with exclusive $\left|V_{c b}\right|$ ), which corresponds to $\left|\varepsilon_{K}\right|_{\mathrm{excl}}^{\mathrm{SM}}$, has $4.2 \sigma$ tension with the experimental result $\left|\varepsilon_{K}\right|^{\operatorname{Exp}}$, while there is no tension in the inclusive $\left|V_{c b}\right|$ channel (heavy quark expansion based on the OPE and QCD sum rules).

In Fig. 5(a), we plot the $\Delta \varepsilon_{K} \equiv\left|\varepsilon_{K}\right|^{\operatorname{Exp}}-\left|\varepsilon_{K}\right|_{\text {excl }}^{\text {SM }}$ in units of $\sigma$ (which is the total error of $\Delta \varepsilon_{K}$ ) as the time evolves starting from 2012. We began to monitor $\Delta \varepsilon_{K}$ in 2012 when several lattice QCD results for $\hat{B}_{K}$ obtained using different discretization methods for the light and strange quarks became consistent with one another within one sigma. In $2012, \Delta \varepsilon_{K}$ was $2.5 \sigma$, but now it is $4.2 \sigma$. To understand the change of $\Delta \varepsilon_{K} / \sigma$ with respect to time, we have performed an additional analysis on the average and error.

In Fig. 5(b), we plot the time evolution of the average $\Delta \varepsilon_{K}$ and the error $\sigma_{\Delta \varepsilon_{K}}$. Here, we find that the average of $\Delta \varepsilon_{K}$ has increased with some fluctuations by $27 \%$ during the period of 2012-2018, and its error $\sigma_{\Delta \varepsilon_{K}}$ has decreased

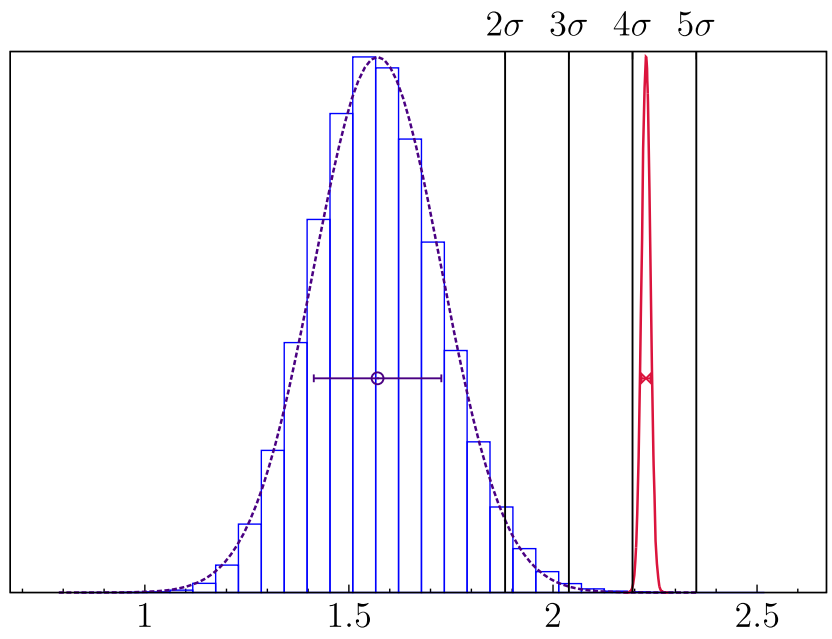

(a) Exclusive $\left|V_{c b}\right|$

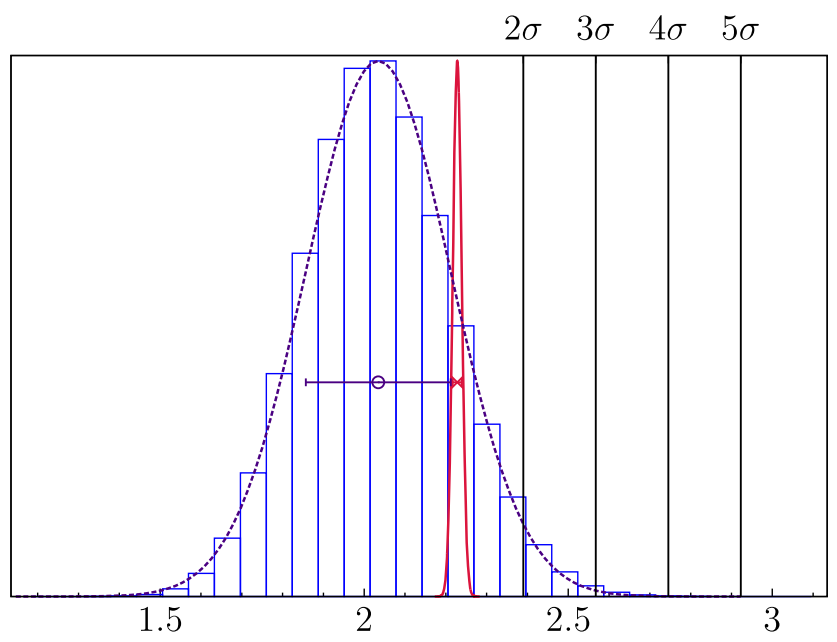

(b) Inclusive $\left|V_{c b}\right|$

FIG. 4. $\left|\varepsilon_{K}\right|$ with (a) exclusive $\left|V_{c b}\right|$ (left) and (b) inclusive $\left|V_{c b}\right|$ (right) in units of $1.0 \times 10^{-3}$. Here, we use the FLAG-2017 $\hat{B}_{K}$, AOF for the Wolfenstein parameters, and the RBC-UKQCD estimate in Eq. (24) for $\xi_{\mathrm{LD}}$. The red curve represents the experimental results for $\varepsilon_{K}$ and the blue curve represents the theoretical results for $\varepsilon_{K}$ calculated directly from the standard model. 


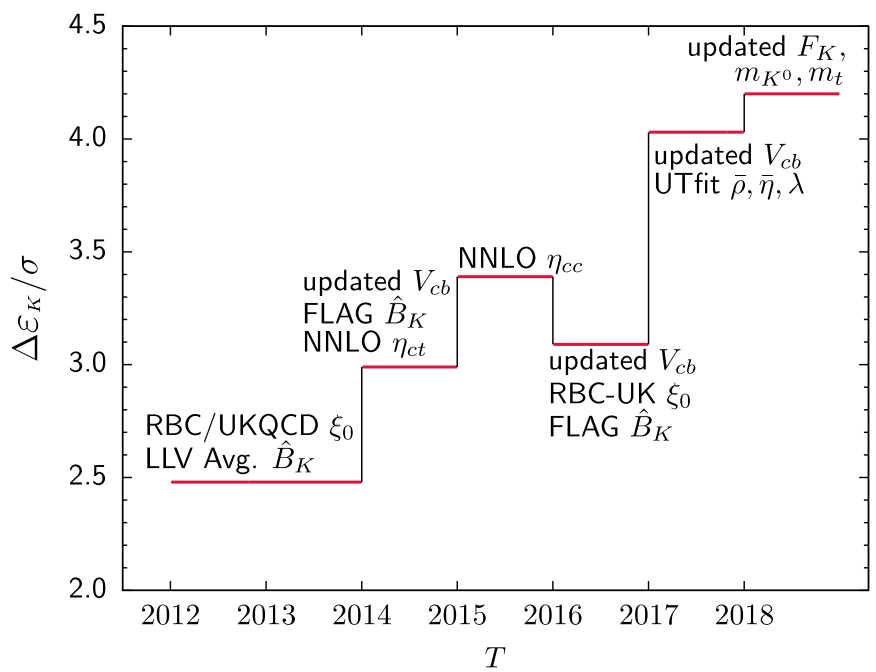

(a) Time evolution of $\Delta \varepsilon_{K} / \sigma$

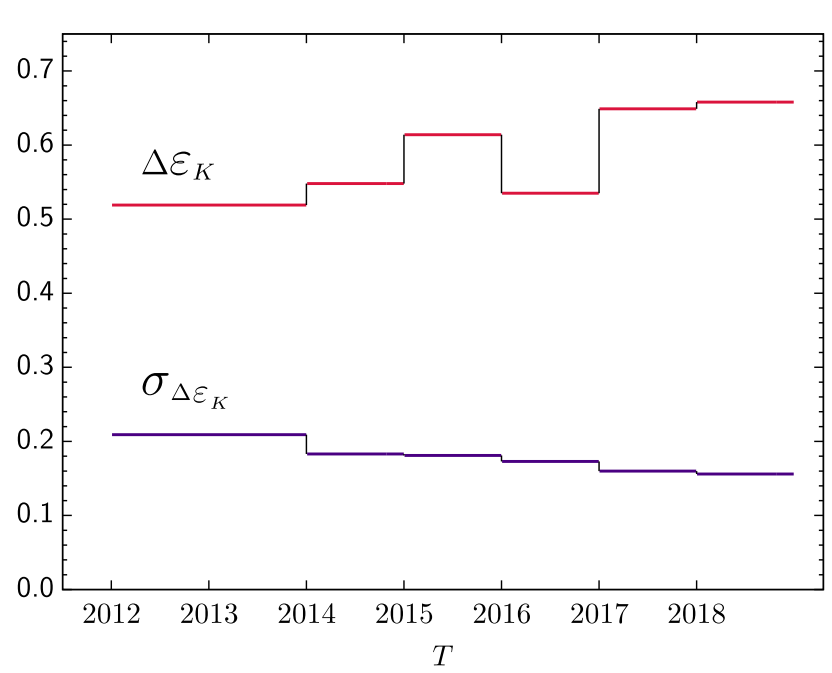

(b) Time evolution of the average and error of $\Delta \varepsilon_{K}$

FIG. 5. Time history of (a) $\Delta \varepsilon_{K} / \sigma$, and (b) $\Delta \varepsilon_{K}$ and $\sigma_{\Delta \varepsilon_{K}}$. We define $\Delta \varepsilon_{K} \equiv\left|\varepsilon_{K}\right|$ Exp $-\left|\varepsilon_{K}\right|_{\text {excl }}^{\text {SM }}$. Here, $\sigma=\sigma_{\Delta \varepsilon_{K}}$ represents the error of $\Delta \varepsilon_{K}$. The $\Delta \varepsilon_{K}$ is obtained using the same input parameters as in Fig. 4(a) for the exclusive $\left|V_{c b}\right|$ channel.

monotonically by $25 \%$ in the same period. These two effects interfere constructively to produce the $4.2 \sigma$ tension in $\Delta \varepsilon_{K}$ in 2018. We can understand the monotonic decrease in $\sigma_{\Delta \varepsilon_{K}}$ in the following way. As time goes on, the lattice QCD calculations are becoming more precise and the experimental results also are becoming more accurate, which constructively leads to the monotonic decrease in $\sigma_{\Delta \varepsilon_{K}}$.

In Table IX, we present the error budget for $\left|\varepsilon_{K}\right|_{\text {excl }}^{\mathrm{SM}}$. Here, we find that the largest error in $\left|\varepsilon_{K}\right|_{\mathrm{excl}}^{\mathrm{SM}}$ comes from $\left|V_{c b}\right|$, while the errors coming from $\bar{\eta}$ and $\eta_{c t}$ are subdominant. Hence, if we are to see a gap $\Delta \varepsilon_{K}$ greater than $5.0 \sigma$, it is essential to reduce the error in $\left|V_{c b}\right|$ significantly.

TABLE IX. Error budget for $\left|\varepsilon_{K}\right|_{\text {excl }}^{\text {SM }}$ obtained using the AOF method for the Wolfenstein parameters, the exclusive $\left|V_{c b}\right|$, the FLAG-2017 $\hat{B}_{K}$, and the RBC-UKQCD estimate for $\xi_{\mathrm{LD}}$. Here, the values are fractional contributions to the total error obtained using the formula in Ref. [5].

\begin{tabular}{lcc}
\hline \hline Source & Error $(\%)$ & Memo \\
\hline$\left|V_{c b}\right|$ & 31.4 & Ex-combined \\
$\bar{\eta}$ & 26.8 & AOF \\
$\eta_{c t}$ & 21.5 & $c-t$ Box \\
$\eta_{c c}$ & 9.1 & $c-c$ Box \\
$\bar{\rho}$ & 4.0 & AOF \\
$\xi_{\mathrm{LD}}$ & 2.5 & $\mathrm{RBC} / \mathrm{UKQCD}$ \\
$\hat{B}_{K}$ & 1.9 & FLAG \\
$\eta_{t t}$ & 0.77 & $t-t$ Box \\
$\xi_{0}$ & 0.70 & $\mathrm{RBC} / \mathrm{UKQCD}$ \\
$m_{t}$ & 0.67 & $m_{t}\left(m_{t}\right)$ \\
$\lambda$ & 0.33 & $\left|V_{u s}\right|$ \\
$\vdots$ & $\vdots$ & $\vdots$ \\
\hline \hline
\end{tabular}

In exclusive $\left|V_{c b}\right|$, there are two major error sources: one is experimental and the other is theoretical. The experimental error is discussed in Sec. III C, and the resolution is beyond the scope of this paper. The largest error in the theoretical part of $\left|V_{c b}\right|$ comes from the heavy quark discretization error (HQDE) for the charm quark in lattice QCD. If one uses the Fermilab action, the HQDE is about $1.0 \%$, which is significantly larger than any other error in the theoretical side. In order to reduce the HQDE by a factor of $\sim 1 / 5$, there are ongoing efforts to use the $\mathrm{OK}$ action to calculate the $B \rightarrow D^{(*)}$ semileptonic form factors in Refs. [65-67].

\section{B. BGI estimate for $\xi_{\mathrm{LD}}$}

Here, we present the results obtained using the BGI estimate in Eq. (23) for $\xi_{\mathrm{LD}}$.

In Fig. 6(a), the blue curve represents the theoretical evaluation of $\left|\varepsilon_{K}\right|$ directly from the SM using the same input parameters as in Fig. 4(a) except for the BGI estimate in Eq. (23) for $\xi_{\mathrm{LD}}$. The red curve in Fig. 6 represents the experimental result for $\left|\varepsilon_{K}\right|$. In Fig. 6(b), the blue curve represents the same as in Fig. 6(a) except for using inclusive $\left|V_{c b}\right|[1 \mathrm{~S}$ scheme in Table III(b)].

Results for $\left|\varepsilon_{K}\right|$ in Fig. 6 are summarized in Table X. From Table $\mathrm{X}$, we find that the value for $\left|\varepsilon_{K}\right|_{\text {excl }}^{\text {SM }}$ (the theoretical evaluation of $\left|\varepsilon_{K}\right|$ with lattice QCD inputs such as exclusive $\left|V_{c b}\right|$ ) has $3.9 \sigma$ tension with the experimental result $\left|\varepsilon_{K}\right|^{\mathrm{Exp}}$, whereas there is no tension in the inclusive $\left|V_{c b}\right|$ channel (with heavy quark expansion and QCD sum rules).

In Fig. 7(a), we plot $\Delta \varepsilon_{K} \equiv\left|\varepsilon_{K}\right|^{\text {Exp }}-\left|\varepsilon_{K}\right|_{\text {excl }}^{\text {SM }}$ in units of $\sigma$ (the total error of $\Delta \varepsilon_{K}$ ) as a function of time starting from 2012. In $2012, \Delta \varepsilon_{K}$ was $2.3 \sigma$, but now it is $3.9 \sigma$. To understand this transition, we have done an additional analysis on the average and error. 


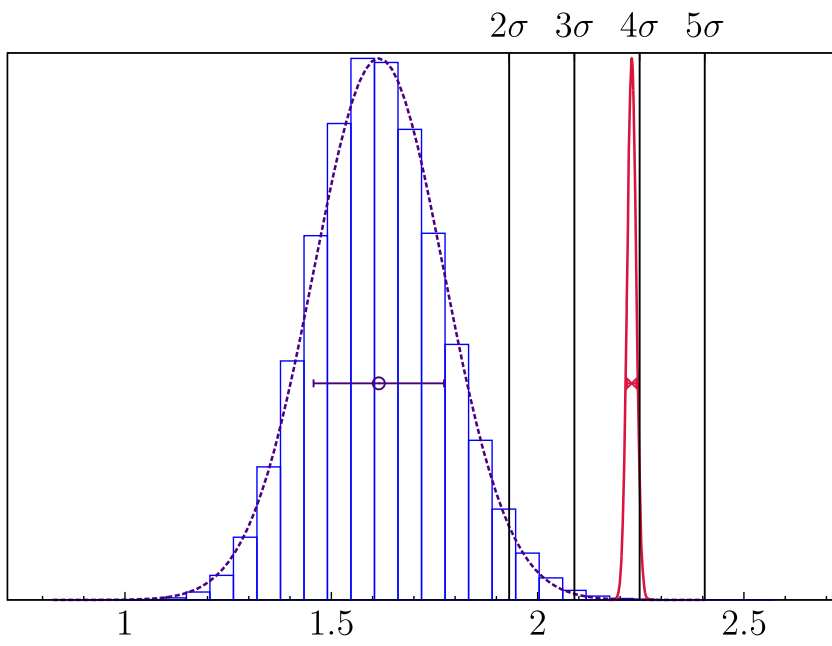

(a) Exclusive $\left|V_{c b}\right|$

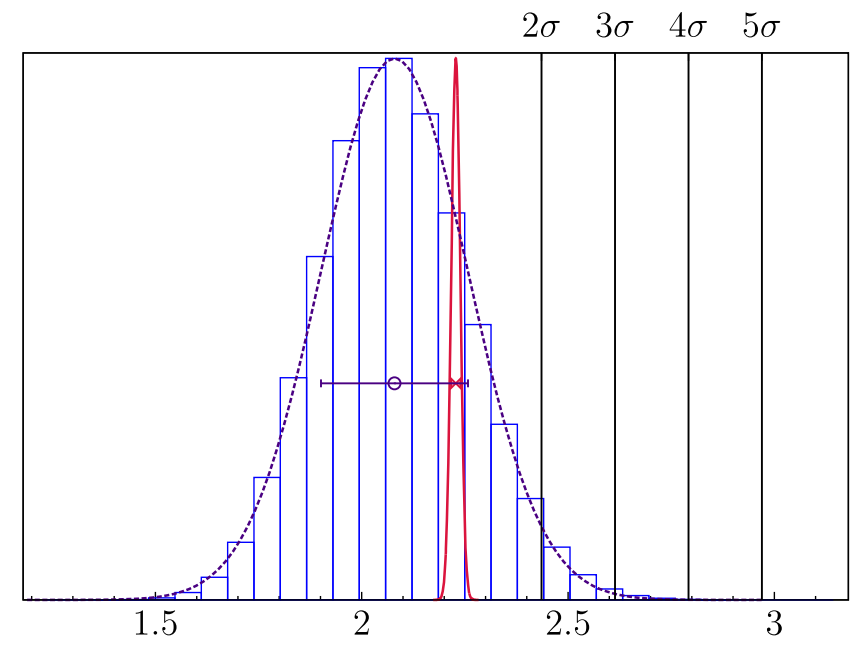

(b) Inclusive $\left|V_{c b}\right|$

FIG. 6. $\left|\varepsilon_{K}\right|$ with (a) exclusive $\left|V_{c b}\right|$ (left) and (b) inclusive $\left|V_{c b}\right|$ (right) in units of $1.0 \times 10^{-3}$. Here, we use the FLAG-2017 $\hat{B}_{K}$, AOF for the Wolfenstein parameters, and the BGI estimate in Eq. (23) for $\xi_{\mathrm{LD}}$. The red curve represents the experimental results for $\varepsilon_{K}$ and the blue curve represents the theoretical results for $\varepsilon_{K}$ calculated directly from the standard model.

TABLE X. $\left|\varepsilon_{K}\right|$ values in units of $1.0 \times 10^{-3}$. The superscripts and subscripts follow the same notation as in Table VIII. We use the same input parameters as in Fig. 6 to determine $\left|\varepsilon_{K}\right|$.

\begin{tabular}{llc}
\hline \hline Parameter & \multicolumn{1}{c}{ Method } & Value \\
\hline$\left|\varepsilon_{K}\right|_{\text {excl }}^{\text {SM }}$ & Exclusive $\left|V_{c b}\right|$ & $1.615 \pm 0.158$ \\
$\left|\varepsilon_{K}\right|_{\text {incl }}^{\text {SM }}$ & Inclusive $\left|V_{c b}\right|$ & $2.079 \pm 0.178$ \\
$\left|\varepsilon_{K}\right|^{\text {Exp }}$ & Experiment & $2.228 \pm 0.011$ \\
\hline \hline
\end{tabular}

In Fig. 7(b), we plot the time evolution of the average and error for $\Delta \varepsilon_{K}$. Here, we find that the average of $\Delta \varepsilon_{K}$ has increased by $29 \%$ with some fluctuations during the period of 2012-2018, and its error has decreased by $25 \%$ monotonically in the same period. These two effects have produced, constructively, the $3.9 \sigma$ tension in $\Delta \varepsilon_{K}$.

In Table XI, we present the error budget for $\left|\varepsilon_{K}\right|_{\text {excl }}^{\text {SM }}$. Here, we find that the largest error in $\left|\varepsilon_{K}\right|_{\text {excl }}^{\text {SM }}$ still comes from $\left|V_{c b}\right|$. Here, note that the error from $\xi_{\mathrm{LD}}$ (the BGI estimate)

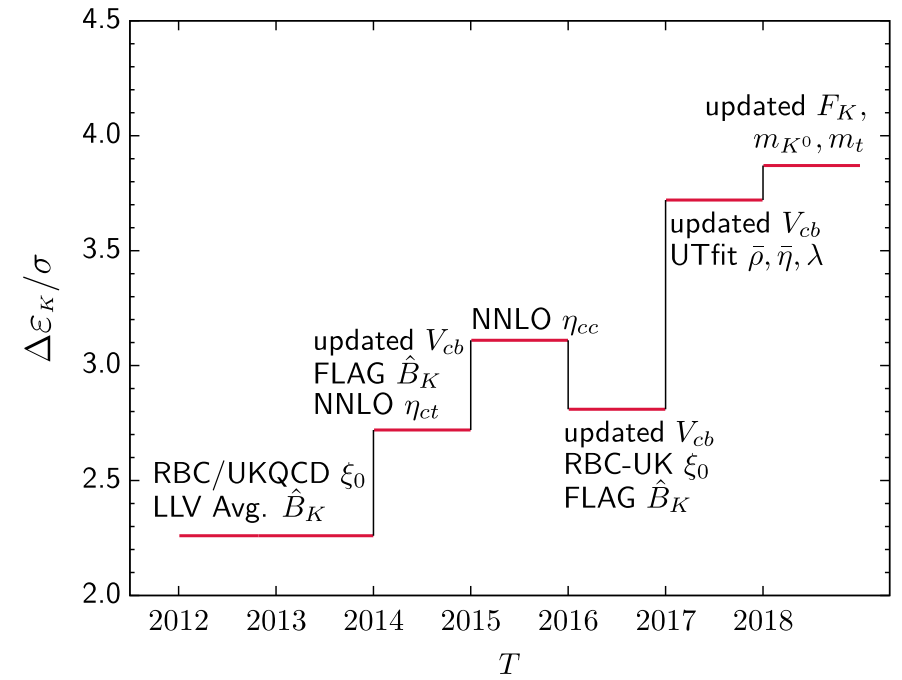

(a) Time evolution of $\Delta \varepsilon_{K} / \sigma$

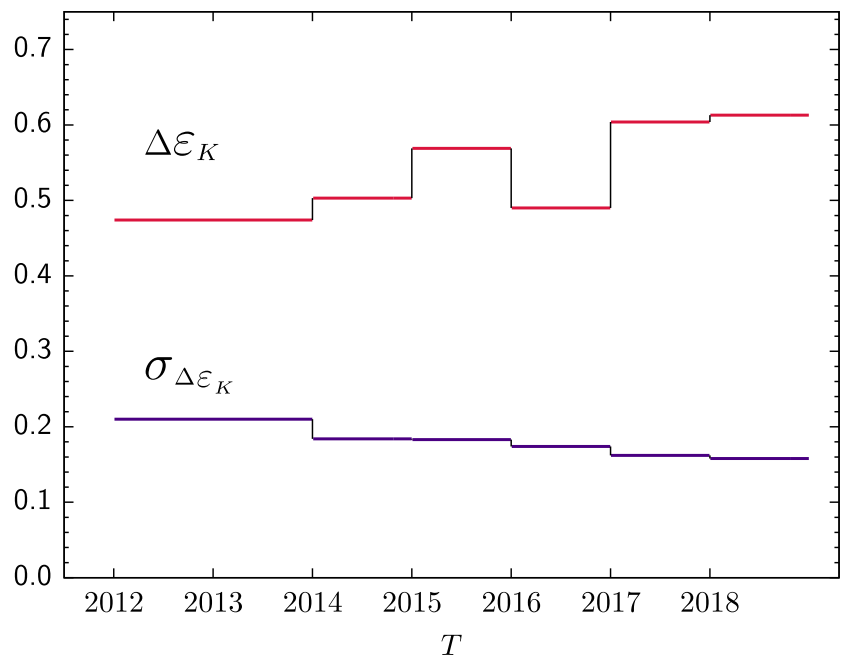

(b) Time evolution of the average and error of $\Delta \varepsilon_{K}$

FIG. 7. Time history of (a) $\Delta \varepsilon_{K} / \sigma$, and (b) $\Delta \varepsilon_{K}$ and $\sigma_{\Delta \varepsilon_{K}}$. We define $\left.\Delta \varepsilon_{K} \equiv\left|\varepsilon_{K}\right|\right|^{\text {Exp }}-\left|\varepsilon_{K}\right|_{\text {excl }}^{\text {SM }}$. Here, $\sigma=\sigma_{\Delta \varepsilon_{K}}$ represents the error of $\Delta \varepsilon_{K}$. The $\Delta \varepsilon_{K}$ is obtained using the same input parameters as in Fig. 6(a) for the exclusive $\left|V_{c b}\right|$ channel. 
TABLE XI. Error budget for $\left|\varepsilon_{K}\right|_{\text {excl }}^{\mathrm{SM}}$ obtained using the AOF method for the Wolfenstein parameters, the exclusive $\left|V_{c b}\right|$, the FLAG-2017 $\hat{B}_{K}$, and the BGI estimate for $\xi_{\mathrm{LD}}$. Here, the values are fractional contributions to the total error obtained using the formula in Ref. [5].

\begin{tabular}{lcc}
\hline \hline Source & Error $(\%)$ & Memo \\
\hline$\left|V_{c b}\right|$ & 30.7 & Ex-combined \\
$\bar{\eta}$ & 26.2 & AOF \\
$\eta_{c t}$ & 21.0 & $c-t$ box \\
$\eta_{c c}$ & 8.9 & $c-c$ box \\
$\xi_{\mathrm{LD}}$ & 4.7 & BGI estimate \\
$\bar{\rho}$ & 3.9 & AOF \\
$\hat{B}_{K}$ & 1.8 & FLAG \\
$\eta_{t t}$ & 0.76 & $t-t$ box \\
$\xi_{0}$ & 0.69 & RBC/UKQCD \\
$m_{t}$ & 0.66 & $m_{t}\left(m_{t}\right)$ \\
$\lambda$ & 0.32 & $\left|V_{u s}\right|$ \\
$\vdots$ & $\vdots$ & $\vdots$ \\
\hline \hline
\end{tabular}

is larger than that from $\bar{\rho}$, which is different from Table IX. In summary, if we are to observe the gap $\Delta \varepsilon_{K}$ greater than $5.0 \sigma$, it is essential to reduce the error in $\left|V_{c b}\right|$ significantly.

\section{CONCLUSION}

In this paper, we find that there exists a remarkable gap of $4.2 \sigma-3.9 \sigma$ in $\varepsilon_{K}$ between experiment and the SM theory with lattice QCD inputs. The upper bound of $4.2 \sigma$ tension is obtained with the RBC-UKQCD estimate for $\xi_{\mathrm{LD}}$. The lower bound of the $3.9 \sigma$ tension is obtained when we use the BGI estimate for $\xi_{\mathrm{LD}}$. In the BGI estimate [60], they added 50\% more error to be on the safe side and more conservative. Even if we remove this $50 \%$ bubble in the error of the BGI estimate, we end up with the same tension of $3.9 \sigma$. To obtain this result, we choose the AOF, exclusive $\left|V_{c b}\right|$ from lattice QCD, and FLAG $\hat{B}_{K}\left(N_{f}=2+1\right)$ from lattice QCD, to determine the theoretical value for $\varepsilon_{K}$ directly from the SM. In 2015, we reported a $3.4 \sigma$ tension between $\left|\varepsilon_{K}\right|_{\text {excl }}^{\mathrm{SM}}$ and $\left.\left|\varepsilon_{K}\right|\right|^{\operatorname{Exp}}$ [5], and the tension is $4.2 \sigma$ at present. ${ }^{4}$ We find that the tension between $\left.\left|\varepsilon_{K}\right|\right|_{\text {excl }} ^{\text {SM }}$ and $\left.\left|\varepsilon_{K}\right|\right|^{\text {Exp }}$ continues to increase during the period of 2012-2018. Part of the reason is that the uncertainties of results for the SM input parameters continue to decrease monotonically.

In Table XII, we present how the values of $\Delta \varepsilon_{K}$ have changed from 2015 to 2018 . Here, we find that the positive shift of $\Delta \varepsilon_{K}$ is about the same for the inclusive and exclusive values of $\left|V_{c b}\right|$. This reflects the changes of other input parameters since 2015. We also note that there is no significant tension observed yet for inclusive $\left|V_{c b}\right|$, which is obtained using the heavy quark expansion based on the QCD sum rules.

\footnotetext{
${ }^{4}$ In 2015 , we used the RBC-UKQCD estimate for $\xi_{\mathrm{LD}}$.
}

TABLE XII. Results for $\Delta \varepsilon_{K}$.

\begin{tabular}{lcc}
\hline \hline Year & Inclusive $\left|V_{c b}\right|$ & Exclusive $\left|V_{c b}\right|$ \\
\hline 2015 & $0.33 \sigma$ & $3.4 \sigma$ \\
2018 & $1.1 \sigma$ & $4.2 \sigma$ \\
\hline \hline
\end{tabular}

There has been an interesting claim $[11,12]$ which has potential to resolve the issue of the inconsistency between the exclusive and inclusive $\left|V_{c b}\right|$. However, this claim is far from conclusive yet since it is based on an analysis over a preliminary and specific subset of experimental data. We find that it would be highly desirable if an experimental group were to perform a comprehensive reanalysis over the entire set of experimental data for the $\bar{B} \rightarrow D^{*} \ell \bar{\nu}$ decays using an alternative parametrization method for the form factors, and compare results with those of CLN.

\section{ACKNOWLEDGMENTS}

We express our sincere gratitude to Carleton Detar, Aida El-Khadra, and Andreas Kronfeld for helpful discussions. We also express sincere gratitude to Guido Martinelli for providing us with the most updated results of UTfit. The research of W. L. is supported by the Creative Research Initiatives Program (Grant No. 2017013332) of the National Research Foundation of Korea (NRF) grant funded by the Korean government (MEST). W. L. acknowledges the support from the KISTI supercomputing center through the strategic support program for the supercomputing application research (Grant No. KSC-2015-G2-0002). Computations were carried out on the DAVID GPU clusters at Seoul National University. J. A. B. is supported by the Basic Science Research Program of the NRF funded by the Ministry of Education (Grant No. 2014027937).

\section{APPENDIX A: BRIEF SUMMARY OF CLN AND BGL}

Let us consider $\bar{B} \rightarrow D^{*} \ell \bar{\nu}$ decays. The recoil variable $w$ is defined as $w \equiv v_{B} \cdot v_{D^{*}}$, where $v_{B}$ is the four velocity of the mother particle ( $\bar{B}$ meson) and $v_{D^{*}}$ is that of the daughter particle ( $D^{*}$ meson). The differential decay rate [68] is given by

$$
\begin{aligned}
\frac{d \Gamma\left(\bar{B} \rightarrow D^{*} \ell \bar{\nu}\right)}{d w}= & \frac{G_{F}^{2} m_{D^{*}}^{3}}{48 \pi^{3}}\left(m_{B}-m_{D^{*}}\right)^{2} \\
& \times \chi(w) \eta_{\mathrm{EW}}^{2} \mathcal{F}^{2}(w)\left|V_{c b}\right|^{2},
\end{aligned}
$$

where $G_{F}$ is Fermi's constant, $\eta_{\mathrm{EW}}$ is a small electroweak correction, and $\mathcal{F}(w)$ is the form factor. The kinematic factor $\chi(w)$ is

$$
\begin{aligned}
\chi(w) & =\sqrt{w^{2}-1}(w+1)^{2} \times Y(w) \\
Y(w) & =\left[1+\frac{4 w}{w+1} \frac{1-2 w r+r^{2}}{(1-r)^{2}}\right],
\end{aligned}
$$


where $r \equiv m_{D^{*}} / m_{B}$. So far the formalism is quite general. We may express the form factor as follows, without loss of generality:

$$
\begin{aligned}
\mathcal{F}^{2}(w)= & h_{A_{1}}^{2}(w) \times \frac{1}{Y(w)} \\
& \times\left\{2 \frac{1-2 w r+r^{2}}{(1-r)^{2}}\left[1+\frac{w-1}{w+1} R_{1}^{2}(w)\right]\right. \\
& \left.+\left[1+\frac{w-1}{1-r}\left(1-R_{2}(w)\right)\right]^{2}\right\} .
\end{aligned}
$$

In the CLN method [30], the form factor functions are parametrized as follows,

$$
\begin{aligned}
h_{A_{1}}(w)= & h_{A_{1}}(1)\left[1-8 \rho^{2} z+\left(53 \rho^{2}-15\right) z^{2}\right. \\
& \left.-\left(231 \rho^{2}-91\right) z^{3}\right] \\
R_{1}(w)= & R_{1}(1)-0.12(w-1)+0.05(w-1)^{2} \\
R_{2}(w)= & R_{2}(1)+0.11(w-1)-0.06(w-1)^{2},
\end{aligned}
$$

where $z$ is a typical conformal mapping variable defined as

$$
z=\frac{\sqrt{w+1}-\sqrt{2}}{\sqrt{w+1}+\sqrt{2}}
$$

The basic idea of CLN is a zero-recoil expansion around $w=1$. The slope and curvature of $R_{1}(w)$ and $R_{2}(w)$ are determined by perturbation theory and $\mathcal{O}(1 / M)$ corrections at the leading order using HQET [68]. The original claim of CLN [30] is that the accuracy of $h_{A_{1}}(w)$ is better than $2 \%$, which makes us become apprehensive as we get $\left|V_{c b}\right|$ in the precision level below $2 \%$. In addition, the slope and curvature of $R_{1}(w)$ and $R_{2}(w)$ contain truncation errors coming from $\mathcal{O}\left(\Lambda^{2} / m_{c}^{2}\right)$ and $\mathcal{O}\left(\alpha_{s} \Lambda / m_{c}\right)$ corrections as well as those uncertainties due to the QCD sum rules on which it is based [11]. Typically, $\Lambda / m_{c} \approx 1 / 3$ and $\alpha_{s} \approx 0.3$, which implies that the accuracy of the slope and curvature in the ratio $R_{i}(w)$ is only in the $10 \%$ level.

Using the CLN method, experimentalists perform a four parameter fit of $\eta_{\mathrm{EW}} \mathcal{F}(1)\left|V_{c b}\right|, \rho^{2}, R_{1}(1)$ and $R_{2}(1)$ to some unfolded data in experiment [10]. Since $\eta_{\mathrm{EW}}$ is very well known and lattice QCD can determine $\mathcal{F}(1)$ very precisely, we can determine $\left|V_{c b}\right|$ from the experimental fits.

Let us switch gears to BGL. In the case of CLN, it is built on the basis of HQET and its perturbative expansion. Unlike CLN, BGL is an HQET-independent approach to the form factor parametrization. The basic idea of BGL is composed of three building blocks: dispersion relationship, analytic continuation, and crossing symmetry.

Let us begin with the first building block: dispersion relation. In QCD, consider the two point function of flavor changing current $J_{\mu}=V_{\mu}, A_{\mu}$, or $(V-A)_{\mu}$, where $V_{\mu}=$ $\bar{c} \gamma_{\mu} b$ and $A_{\mu}=\bar{c} \gamma_{\mu} \gamma_{5} b$.

$$
\begin{aligned}
\Pi_{J}^{\mu \nu}(q) & =\left(q^{\mu} q^{\nu}-q^{2} g^{\mu \nu}\right) \Pi_{J}^{T}\left(q^{2}\right)+g^{\mu \nu} \Pi_{J}^{L}\left(q^{2}\right) \\
& \equiv i \int d^{4} x e^{i q \cdot x}\left\langle 0\left|T J^{\mu}(x)\left[J^{\nu}(0)\right]^{\dagger}\right| 0\right\rangle .
\end{aligned}
$$

In general, $\Pi_{J}^{T, L}\left(q^{2}\right)$ is not finite. Hence, in order to obtain finite dispersion relations, we need to make one or two subtractions as follows,

$$
\begin{aligned}
& \chi_{J}^{L}\left(q^{2}\right)=\frac{\partial \Pi_{J}^{L}}{\partial q^{2}}=\frac{1}{\pi} \int_{0}^{\infty} d t \frac{\operatorname{Im} \Pi_{J}^{L}(t)}{\left(t-q^{2}\right)^{2}} \\
& \chi_{J}^{T}\left(q^{2}\right)=\frac{\partial \Pi_{J}^{T}}{\partial q^{2}}=\frac{1}{\pi} \int_{0}^{\infty} d t \frac{\operatorname{Im} \Pi_{J}^{T}(t)}{\left(t-q^{2}\right)^{2}} .
\end{aligned}
$$

Let us introduce the Källen-Lehmann spectral decomposition by inserting a complete set of states $X$ into the two point function.

$$
\begin{aligned}
& \left(q^{\mu} q^{\nu}-q^{2} g^{\mu \nu}\right) \operatorname{Im} \Pi_{J}^{T}\left(q^{2}\right)+g^{\mu \nu} \operatorname{Im} \Pi_{J}^{L}\left(q^{2}\right) \\
& \quad=\frac{1}{2} \sum_{X}(2 \pi)^{4} \delta^{4}\left(q-p_{X}\right)\left\langle 0\left|J^{\mu}(0)\right| X\right\rangle\left\langle X\left|\left[J^{\nu}(0)\right]^{\dagger}\right| 0\right\rangle,
\end{aligned}
$$

where the sum includes an integral over the phase space allowed for each state $X$ which has the same quantum number as the current $J$. The positivity of $\operatorname{Im} \Pi_{J}^{T}\left(q^{2}\right)$ and $\operatorname{Im} \Pi_{J}^{L}\left(q^{2}\right)$ follows from Eq. (A12) [39]. In other words,

$\left[\left(q^{\mu} q^{\nu}-q^{2} g^{\mu \nu}\right) \operatorname{Im} \Pi_{J}^{T}\left(q^{2}\right)+g^{\mu \nu} \operatorname{Im} \Pi_{J}^{L}\left(q^{2}\right)\right] \xi_{\mu} \xi_{\nu}^{*} \geq 0$

for any complex 4-vector $\xi_{\mu}$. This implies that

$$
\begin{gathered}
\operatorname{Im} \Pi_{J}^{T}\left(q^{2}\right) \geq 0 \\
\operatorname{Im} \Pi_{J}^{L}\left(q^{2}\right) \geq 0 .
\end{gathered}
$$

Let us consider $\operatorname{Im} \prod_{J}^{i i}\left(q^{2}\right)$ in Eq. (A12) (no sum in $i$ index).

$$
\begin{aligned}
\operatorname{Im} \Pi_{J}^{i i}\left(q^{2}\right)= & \frac{1}{2} \int \frac{d^{3} p_{1} d^{3} p_{2}}{(2 \pi)^{3} 4 E_{1} E_{2}} \delta^{4}\left(q-p_{1}-p_{2}\right) \\
& \times \sum_{\mathrm{pol}}\left\langle 0\left|J^{i}\right| H_{b}\left(p_{1}\right) H_{c}\left(p_{2}\right)\right\rangle \\
& \times\left\langle H_{b}\left(p_{1}\right) H_{c}\left(p_{2}\right)\left|\left[J^{i}\right]^{\dagger}\right| 0\right\rangle+\cdots,
\end{aligned}
$$

where the sum is over polarizations of $H_{b}$ and $H_{c}$ states, and the ellipsis denotes strictly positive contributions from the higher resonances and multiparticle states (three-body 
or higher multibody states). Here, we assume that $H_{b}=$ $B, B^{*}$ meson states, and $H_{c}=D, D^{*}$ meson states. Since the right-hand side of Eq. (A16) is a sum of positive contributions, we can obtain the following simple inequality:

$$
\operatorname{Im} \Pi_{J}^{i i}(t) \geq k(t)|F(t)|^{2},
$$

where $t=q^{2}, k(t)$ is a calculable kinematic function arising from two-body phase space, and $F(t)$ is the form factor associated with a specific decay of our interest. For example, in the case of $\bar{B} \rightarrow D^{*} \ell \bar{\nu}$ decay channel,

$$
\begin{aligned}
k(t)|F(t)|^{2}= & \frac{1}{2} \int \frac{d^{3} p_{1} d^{3} p_{2}}{(2 \pi)^{3} 4 E_{1} E_{2}} \delta^{4}\left(q-p_{1}-p_{2}\right) \\
& \times \sum_{\mathrm{pol}}\left\langle 0\left|J^{i}\right| B\left(p_{1}\right) D^{*}\left(p_{2}\right)\right\rangle \\
& \times\left\langle B\left(p_{1}\right) D^{*}\left(p_{2}\right)\left|\left[J^{i}\right]^{\dagger}\right| 0\right\rangle .
\end{aligned}
$$

At this stage, we need to use the second building block: crossing symmetry [33]. Let us define $t_{ \pm} \equiv\left(M_{H_{b}} \pm M_{H_{c}}\right)^{2}$. The crossing symmetry insures that the $\left\langle 0\left|J^{i}\right| H_{b} H_{c}\right\rangle$ amplitude which shows up in pair production of the $H_{b}$ and $H_{c}$ mesons from a virtual $W$ boson shares the same form factor $F(t)$ as the $\left\langle\bar{H}_{c}\left|J^{i}\right| H_{b}\right\rangle$ amplitude, while we can connect the pair-production region $t_{+} \leq t<\infty$ with the semileptonic region $m_{\ell}^{2} \leq t \leq t_{-}$through the analytic continuation (the third building block).

Let us define the hadronic moments $\chi_{J}^{(n)}$ as in Refs. [33,34],

$$
\begin{aligned}
\left.\chi_{J} \equiv \frac{1}{2} \frac{\partial^{2} \Pi_{J}^{i i}}{\partial^{2} q^{2}}\right|_{q^{2}=0} & =\left[\frac{\partial \Pi_{J}^{T}}{\partial q^{2}}-\frac{1}{2} \frac{\partial^{2} \Pi_{J}^{L}}{\partial^{2} q^{2}}\right]_{q^{2}=0} \\
& =\left[\chi_{J}^{T}\left(q^{2}\right)-\frac{1}{2} \frac{\partial \chi_{J}^{L}}{\partial q^{2}}\right]_{q^{2}=0} \\
& =\left.\frac{1}{\pi} \int_{0}^{\infty} d t \frac{\operatorname{Im} \Pi_{J}^{i i}(t)}{\left(t-q^{2}\right)^{3}}\right|_{q^{2}=0} \\
\chi_{J}^{(n)} & \left.\equiv \frac{1}{\Gamma(n+3)} \frac{\partial^{n+2} \Pi_{J}^{i i}}{\partial^{n+2} q^{2}}\right|_{q^{2}=0} \\
& =\left.\frac{1}{\pi} \int_{0}^{\infty} d t \frac{\operatorname{Im} \Pi_{J}^{i i}(t)}{\left(t-q^{2}\right)^{n+3}}\right|_{q^{2}=0},
\end{aligned}
$$

where $\chi_{J}^{(0)}=\chi_{J}$. Hence, from the inequality in Eq. (A17), we can obtain the following inequality:

$$
\chi_{J}^{(n)} \geq \frac{1}{\pi} \int_{t_{+}}^{\infty} d t \frac{k(t)|F(t)|^{2}}{t^{n+3}},
$$

where $t_{+}$represents the pair-production threshold.

At this stage, we need to introduce a key idea of quarkhadron duality in QCD sum rules which claims that the hadronic moments $\chi_{J}^{(n)}$ can be calculated in perturbative $\mathrm{QCD}$ at $q^{2}=0$ [34]. Then, we can rewrite the inequality in Eq. (A21) as follows,

$$
\frac{1}{\pi} \int_{t_{+}}^{\infty} d t\left|h^{(n)}(t) F(t)\right|^{2} \leq 1
$$

where

$$
\left[h^{(n)}(t)\right]^{2}=\frac{k(t)}{t^{n+3} \chi_{J}^{(n)}} \geq 0
$$

The inequality in Eq. (A22) imposes an upper bound on the form factor $F(t)$ in the pair-production region $\left(t_{+} \leq t<\infty\right)$.

In order to turn Eq. (A22) into a constraint in the semileptonic region $\left(m_{\ell}^{2} \leq t \leq t_{-}\right)$, we need to use the third building block: analyticity which allows us to extend the analytic region of the integrand to the region below the pair-production threshold $\left(t<t_{+}\right)$. To do this, it is convenient to introduce a conformal mapping function,

$$
z\left(t, t_{s}\right)=\frac{\sqrt{t_{+}-t}-\sqrt{t_{+}-t_{s}}}{\sqrt{t_{+}-t}+\sqrt{t_{+}-t_{s}}} .
$$

$z$ is real for $t<t_{+}, z=-1$ at $t=t_{+}, 0$ at $t=t_{s}$, and a $\mathrm{U}(1)$ phase $z=e^{i \theta}$ for $t>t_{+} \cdot t_{s} \geq t>-\infty$ maps into $0 \leq z<1$ along the real axis. The upper contour of $t_{+} \leq t<\infty$ along the real axis maps into the upper half of a unit circle $\left(\pi \geq \theta>0\right.$ for $\left.z=e^{i \theta}\right)$. Similarly, the lower contour of $t_{+} \leq t<\infty$ along the real axis maps into the lower half of a unit circle $\left(\pi \leq \theta<2 \pi\right.$ for $\left.z=e^{i \theta}\right)$.

All the poles in the integrand of Eq. (A22) can be removed by multiplying by various powers of $z\left(t, t_{s}\right)$, if we know the positions $t_{s}$ of the subthreshold poles in $F(t)$ and $h^{(n)}(t)$. Each pole has a distinct value of $t_{s}$, and the product $z\left(t, t_{s 1}\right) z\left(t, t_{s 2}\right) z\left(t, t_{s 3}\right) \cdots$ can remove all of them. For example, such poles include the contribution of $B_{c}$ resonances to the form factor $F(t)$ as well as singularities in the kinematic function $h^{(n)}(t)$.

Once we determine the pole positions phenomenologically, we can rewrite the inequality in Eq. (A22) as follows,

$$
\frac{1}{\pi} \int_{t_{+}}^{\infty} d t\left|\frac{d z\left(t, t_{0}\right)}{d t}\right|\left|\phi\left(t, t_{0}\right) P(t) F(t)\right|^{2} \leq 1,
$$

where the outer function $\phi$ is

$$
\phi\left(t, t_{0}\right)=\tilde{P}(t) \frac{h^{(n)}(t)}{\sqrt{\left|\frac{d z\left(t, t_{0}\right)}{d t}\right|}}
$$


The factor $\tilde{P}(t)$ is a product of $z\left(t, t_{s}\right)$ 's and $\sqrt{z\left(t, t_{s}\right)}$ 's such that $t_{s}$ is chosen to remove the subthreshold poles and branch cuts in the kinematic function $h^{(n)}(t)$. The Blaschke factor $P(t)$ is

$$
\begin{aligned}
P(t) & \equiv \prod_{i=1}^{N} \frac{z-z_{P_{i}}}{1-z z_{P_{i}}^{*}}=\prod_{i=1}^{N} \frac{z-z_{P_{i}}}{1-z z_{P_{i}}} \\
z_{P_{i}} & \equiv z\left(t_{P_{i}}, t_{-}\right)=\frac{\sqrt{t_{+}-t_{P_{i}}}-\sqrt{t_{+}-t_{-}}}{\sqrt{t_{+}-t_{P_{i}}}+\sqrt{t_{+}-t_{-}}},
\end{aligned}
$$

where $t_{P_{i}}=M_{P_{i}}^{2}$ represents the pole positions of $F(t)$ below the threshold $\left(t_{P_{i}}<t_{+}\right)$, and $N$ is the number of the subthreshold poles in $F(t)$. Here, note that $z_{P_{i}}$ is real $\left(z_{P_{i}}^{*}=z_{P_{i}}\right)$ for the subthreshold poles. In addition, note that $P(t)$ is unimodular $(|P(t)|=1)$, if $z$ is unimodular $(|z|=1)$. We have full freedom to choose $t_{0}$. Here, we set $t_{0}=t_{-}$for convenience and simplicity, and without loss of generality. ${ }^{5}$

Since $\phi\left(t, t_{0}\right) P(t) F(t)$ is analytic even in the subthreshold region, it is possible to expand this in powers of $z\left(t, t_{0}\right)$. Hence,

$$
F(t)=\frac{1}{\phi\left(t, t_{0}\right) P(t)} \sum_{n=0}^{\infty} a_{n} z^{n}\left(t, t_{0}\right)
$$

This is called the BGL method of form factor parametrization [32]. In addition, the integral in Eq. (A25) can be rewritten as follows,

$$
\frac{1}{\pi} \int_{t_{+}}^{\infty} d t\left|\frac{d z\left(t, t_{0}\right)}{d t}\right|=\frac{1}{2 \pi} \int_{0}^{2 \pi} d \theta
$$

where $z=e^{i \theta}$ above the threshold $\left(t_{+} \leq t<\infty\right)$. Hence, the final version of the inequality after the Fourier analysis is

$$
\sum_{n=0}^{\infty} a_{n}^{2} \leq 1
$$

This is called the unitarity condition (the weak version). ${ }^{6}$

For the $\bar{B} \rightarrow D^{*} \ell \bar{\nu}$ decay process that is the main subject of this paper, $z\left(t, t_{0}\right)$ is in the physical region of $0 \leq z \leq$ 0.056 for any physical momentum transfer $m_{\ell}^{2} \leq t \leq t_{-}$. Hence, in practice, it is possible to truncate the expansion after the first two or three terms. Since the BGL method does not use any model to constrain $a_{n}$, it is model independent by construction.

\footnotetext{
${ }^{5}$ For other choices of $t_{0}$, refer to Ref. [34].

${ }^{6} \mathrm{~A}$ stronger version can be obtained simply by adding more decay channels in the right-hand side of the inequality in Eq. (A17) [11].
}

\section{APPENDIX B: CONVERSION FORMULA OF THE TOP POLE QUARK MASS TO THE TOP $\overline{M S}$ QUARK MASS}

\section{The scale-invariant mass}

We follow the terminology in the literature. The pole and $\overline{\mathrm{MS}}$ masses are related by the ratio of (mass) renormalization factors $z$,

$$
\frac{m(\mu)}{M}=z(\mu) \equiv \frac{Z_{\mathrm{OS}}}{Z_{\overline{\mathrm{MS}}}},
$$

where $m(\mu)$ is the $\overline{\mathrm{MS}}$ mass renormalized at scale $\mu, M$ is the pole mass, $Z_{\mathrm{OS}}$ is the renormalization factor in the onshell scheme, and $Z_{\overline{\mathrm{MS}}}$ is the renormalization factor in the $\overline{\mathrm{MS}}$ scheme. The ratio $z$ depends on the scale $\mu$ via the strong coupling $\alpha_{s}(\mu)$ and $\ln (\mu / M)$. The top SI mass $\mu_{t}$ is the $\overline{\mathrm{MS}}$ mass $m_{t}(\mu)$ with the scale $\mu$ set equal to the scaleinvariant mass,

$$
\mu_{t} \equiv m_{t}\left(\mu_{t}\right)
$$

Given the perturbative expansion of $z(\mu)$ in powers of $\alpha_{s}(\mu)$, Eq. (B2) gives the SI mass in terms of $\alpha_{s}\left(\mu_{t}\right)$ and $\ln \left(\mu_{t} / M_{t}\right)$, i.e., in terms of the SI mass and the pole mass. To obtain a formula for the SI mass in terms of the pole mass alone, one can iterate the perturbative expansion. The result is an expansion of $\mu_{t}$ in powers of $\alpha_{s}\left(M_{t}\right)$.

\section{Three-loop result}

For the three-loop conversion, we use Eq. (16) of Ref. [69]. For the top mass conversion, this equation is an expansion of the ratio $z\left(\mu_{t}\right)$ in powers of the coupling $\alpha_{s}^{(6)}\left(M_{t}\right)$, with six active quark flavors. We obtain the coupling $\alpha_{s}^{(6)}\left(M_{t}\right)$ by running the five-flavor coupling $\alpha_{s}^{(5)}\left(M_{Z}\right)$ at the $Z$-boson mass to the five-flavor coupling $\alpha_{s}^{(5)}\left(M_{t}\right)$ at the top pole and then matching across threshold. The parameter $n_{l}$ is the number of light quarks; for the top mass conversion, $n_{l}=5$. The quantity $\Delta\left(M_{i} / M\right)$ is the two-loop mass correction for a light quark with pole mass $M_{i} ; \Delta(0)=0$. The three-loop coefficient $z_{m}^{\text {SI,(3) }}(M)$ can be found by iterating Eq. (13) of Ref. [69] and using Eq. (15). We have verified that doing so yields results consistent with Refs. [69,70] and the RunDec3 code [71]. Our result for the top SI mass is

$$
\mu_{t}=163.82 \pm 1.05 \mathrm{GeV},
$$

where the uncertainty is propagated from the uncertainty in the top pole mass, and all other uncertainties are neglected. Below we detail the inputs and the steps of the calculation. 
For the running we use Eq. (5) of Ref. [69], which is the four-loop solution to the renormalization group equations, expressed in terms of $\ln (\mu / \Lambda)$, where $\Lambda$ is the QCD scale. To obtain $\alpha_{s}^{(5)}\left(M_{t}\right)=0.107660$, we set $\mu=M_{t}=173.5 \pm$ $1.1 \mathrm{GeV}$ [3] and fix $\ln \left(M_{t} / \Lambda\right)$ using Eq. (4) of Ref. [69], with $\alpha_{s}^{(5)}\left(M_{Z}\right)=0.1181$ [3] and $M_{Z}=91.1876 \mathrm{GeV}$ [3]. We find $\Lambda=209.78 \mathrm{MeV}$, in agreement with Table II of Ref. [71]. To obtain $\alpha_{s}^{(6)}\left(M_{t}\right)=0.107714$, we use the decoupling relation in Eqs. (19) and (25) of Ref. [69], which is the three-loop expansion of $\alpha_{s}^{(6)}\left(M_{t}\right)$ in powers of $\alpha_{s}^{(5)}\left(M_{t}\right)$.

We set all light-quark mass corrections to 0 except that for the bottom quark. For the bottom quark pole mass, we use Eq. (71.21) of Ref. [3], the three-loop expansion of the pole mass, with $\mu_{b}=m_{b}\left(\mu_{b}\right)=4.18 \mathrm{GeV}$ to obtain $M_{b}=4.9324 \mathrm{GeV}$. Then Eq. (19) of Ref. [72] yields $\Delta\left(M_{b} / M_{t}\right)=0.0344909$.

We obtain the three-loop coefficient $z_{m}^{\mathrm{SI},(3)}(M)$ in terms of the coefficients in the expansion of $z(\mu)$ in powers of $\alpha_{s}(\mu)$,

$$
\begin{aligned}
z(\mu)= & 1+\frac{\alpha_{s}(\mu)}{\pi} z_{1}(\mu)+\left(\frac{\alpha_{s}(\mu)}{\pi}\right)^{2} z_{2}(\mu) \\
& +\left(\frac{\alpha_{s}(\mu)}{\pi}\right)^{3} z_{3}(\mu) .
\end{aligned}
$$

The coefficients $z_{1}(\mu), z_{2}(\mu)$, and $z_{3}(\mu)$ are given explicitly in Eqs. (13) and (15) of Ref. [69]. Taylor expanding the corresponding terms in Eq. (B4) about the pole mass $M$, using the definition of the beta function, setting $m(\mu)=\mu$, and iteratively solving the result for $\mu / M$ yields

$$
\begin{aligned}
\frac{\mu}{M}= & 1+\frac{\alpha_{s}(M)}{\pi} z_{m}^{\mathrm{SI},(1)}(M)+\left(\frac{\alpha_{s}(M)}{\pi}\right)^{2} z_{m}^{\mathrm{SI},(2)}(M) \\
& +\left(\frac{\alpha_{s}(M)}{\pi}\right)^{3} z_{m}^{\mathrm{SI},(3)}(M),
\end{aligned}
$$

where

$$
\begin{aligned}
z_{m}^{\mathrm{SI},(1)}(M)= & z_{1}(M), \\
z_{m}^{\mathrm{SI},(2)}(M)= & z_{2}(M)+z_{1}(M) M z_{1}^{\prime}(M), \\
z_{m}^{\mathrm{SI},(3)}(M)= & z_{3}(M)+z_{2}(M) M z_{1}^{\prime}(M)+z_{1}(M) M z_{2}^{\prime}(M) \\
& -2 \beta_{0} z_{1}^{2}(M)+z_{1}(M)\left(M z_{1}^{\prime}(M)\right)^{2} \\
& +\frac{1}{2}\left(z_{1}(M)\right)^{2} M^{2} z_{1}^{\prime \prime}(M) .
\end{aligned}
$$

Primes denote derivatives with respect to the scale $\mu$, all coefficients are evaluated at $\mu=M$, the leading order betafunction coefficient is that for the six-flavor coupling, and to obtain agreement with the literature, we neglect the twoloop fermion mass correction when calculating the threeloop coefficient.

\section{Four-loop result}

For the four-loop conversion, we consider the generalization of Eq. (16) of Ref. [69] to four loops. We use five-loop running [73] and four-loop matching [74] to obtain the coupling $\alpha_{s}^{(6)}\left(M_{t}\right)$. For the four-loop coefficient $z_{m}^{\mathrm{SI}(4)}(M)$, we verify that the numerical expression in the RunDec3 code [71] agrees with the literature [75]. The three-loop fermion mass correction is known to be somewhat larger than the two-loop correction $[71,76]$, but we neglect it. Our result for the SI mass is

$$
\mu_{t}=163.65 \pm 1.05 \mathrm{GeV}
$$

where the uncertainty is again that propagated from the pole mass. Again, we neglect all other sources of error. Below we provide details.

The inputs are the same as for the three-loop calculation. The extension of Eqs. (4) and (5) of Ref. [69] to five-loop order is in the RunDec3 code [71]. For the QCD scale, we find $\Lambda=209.80 \mathrm{MeV}$, in agreement with Table II of Ref. [71], and for the five-flavor coupling, we find $\alpha_{s}^{(5)}\left(M_{t}\right)=0.107643$. We match the coupling across threshold using Eq. (19) of Ref. [69] with the decoupling factor from Eqs. (54), (59)-(63), (19), and (20) of Ref. [74]. We find $\alpha_{s}^{(6)}\left(M_{t}\right)=0.107703$.

To compare the coefficient $z_{m}^{\text {SI, (4) }}(M)$ from the RunDec3 code with the literature, we begin with $z_{4}(M)$, which enters the expansion of $z(M)$ at four loops [cf. Eq. (B4)],

$$
\begin{aligned}
z(M)= & 1+\frac{\alpha_{s}(M)}{\pi} z_{1}(M)+\left(\frac{\alpha_{s}(M)}{\pi}\right)^{2} z_{2}(M) \\
& +\left(\frac{\alpha_{s}(M)}{\pi}\right)^{3} z_{3}(M)+\left(\frac{\alpha_{s}(M)}{\pi}\right)^{4} z_{4}(M) .
\end{aligned}
$$

We obtain a numerical result for $z_{4}(M)$ from Eqs. (15) and (23) of Ref. [75]. This result agrees with that in the RunDec3 code. We then relate $z_{4}(M)$ to $z_{m}^{\mathrm{SI},(4)}(M)$ by iterating the expansion of $z(\mu)$ for $m(\mu)=\mu$ to obtain the expansion of $\mu / M$ in powers of $\alpha_{s}(M)$. We have done this calculation twice, once numerically and once analytically. The relation between $z_{4}(M)$ and $z_{m}^{\mathrm{SI},(4)}(M)$ is 


$$
\begin{aligned}
z_{m}^{\mathrm{SI},(4)}(M)= & z_{4}(M)+z_{1}(M) M^{2} z_{1}^{\prime \prime}(M)\left(z_{2}(M)+z_{1}(M) M z_{1}^{\prime}(M)\right) \\
& +\left(z_{1}(M)\right)^{2}\left(\frac{1}{2} M^{2} z_{2}^{\prime \prime}(M)-2 \beta_{0} M z_{1}^{\prime}(M)+\beta_{0} z_{1}(M)\right) \\
& +\frac{1}{6}\left(z_{1}(M)\right)^{3} M^{3} z_{1}^{\prime \prime \prime}(M)+z_{1}(M)\left(M z_{3}^{\prime}(M)-4 \beta_{0} z_{2}(M)\right) \\
& +\left(z_{2}(M)+z_{1}(M) M z_{1}^{\prime}(M)\right)\left(M z_{2}^{\prime}(M)-2 \beta_{0} z_{1}(M)\right) \\
& +M z_{1}^{\prime}(M)\left(z_{3}(M)+\frac{1}{2}\left(z_{1}(M)\right)^{2} M^{2} z_{1}^{\prime \prime}(M)+z_{1}(M)\right. \\
& \left.\times\left(M z_{2}^{\prime}(M)-2 \beta_{0} z_{1}(M)\right)+M z_{1}^{\prime}(M)\left(z_{2}(M)+z_{1}(M) M z_{1}^{\prime}(M)\right)\right) \\
& -2 \beta_{1}\left(z_{1}(M)\right)^{2} .
\end{aligned}
$$

Both our calculations yield agreement with the result for $z_{m}^{\mathrm{SI},(4)}(M)$ in the RunDec3 code, provided that the coefficient $z_{3}(\mu)$ given in Eq. (13) of Ref. [69] is incorrect: The first $\left(\ln \mu^{2} / M^{2}\right)^{2}$ appearing in the $n_{l}^{2}$ term there should be $\ln \left(\mu^{2} / M^{2}\right)$. This evident typo in Ref. [69] does not affect the three-loop conversion because no derivatives of $z_{3}(\mu)$ enter.

[1] G. Buchalla, A. J. Buras, and M. E. Lautenbacher, Rev. Mod. Phys. 68, 1125 (1996).

[2] A. J. Buras, CERN Report No. TUM-HEP-316, 1998.

[3] C. Patrignani et al. (Particle Data Group), Chin. Phys. C 40, 100001 (2016).

[4] X. Feng, EPJ Web Conf. 175, 01005 (2018).

[5] J. A. Bailey, Y.-C. Jang, W. Lee, and S. Park (SWME Collaboration), Phys. Rev. D 92, 034510 (2015).

[6] S. Aoki et al., Eur. Phys. J. C 77, 112 (2017).

[7] Y.-C. Jang, W. Lee, S. Lee, and J. Leem (SWME Collaboration), EPJ Web Conf. 175, 14015 (2018).

[8] W. Lee, J. Phys. Conf. Ser. 800, 012006 (2017).

[9] J. A. Bailey, W. Lee, J. Leem, S. Park, and Y.-C. Jang, Proc. Sci. LATTICE2016 (2016) 383 [arXiv:1611.00503].

[10] Y. Amhis et al. (HFLAV Collaboration), Eur. Phys. J. C 77, 895 (2017).

[11] D. Bigi, P. Gambino, and S. Schacht, Phys. Lett. B 769, 441 (2017).

[12] B. Grinstein and A. Kobach, Phys. Lett. B 771, 359 (2017).

[13] T. Inami and C. Lim,Prog. Theor. Phys. 65, 297 (1981).

[14] S. Herrlich and U. Nierste, Nucl. Phys. B476, 27 (1996).

[15] J. Charles, A. Höcker, H. Lacker, S. Laplace, F. R. Diberder, J. Malclés, J. Ocariz, M. Pivk, and L. Roos (CKMfitter Group), Eur. Phys. J. C 41, 1 (2005), updated results and plots available at: http://ckmfitter.in2p3.fr.

[16] M. Bona et al. (UTfit Collaboration), J. High Energy Phys. 10 (2006) 081; Standard Model fit results: Summer 2016 (ICHEP 2016): http://www.utfit.org.

[17] B. Winstein and L. Wolfenstein, Rev. Mod. Phys. 65, 1113 (1993).

[18] A. Bevan et al., Nucl. Phys. B, Proc. Suppl. 241-242, 89 (2013).

[19] G. Martinelli et al., Private communication with UTfit, http://www.utfit.org/UTfit/ (2017).
[20] N. Carrasco, P. Dimopoulos, R. Frezzotti, V. Lubicz, G. C. Rossi, S. Simula, and C. Tarantino (ETM Collaboration), Phys. Rev. D 92, 034516 (2015).

[21] N. H. Christ, T. Izubuchi, C. T. Sachrajda, A. Soni, and J. Yu (RBC and UKQCD Collaborations), Phys. Rev. D 88, 014508 (2013).

[22] Z. Bai, N. Christ, T. Izubuchi, C. Sachrajda, A. Soni, and J. Yu, Phys. Rev. Lett. 113, 112003 (2014).

[23] S. Durr et al., Phys. Lett. B 705, 477 (2011).

[24] J. Laiho and R. S. Van de Water, Proc. Sci. LATTICE2011 (2011) 293 [arXiv:1112.4861].

[25] T. Blum et al. (RBC and UKQCD Collaboration), Phys. Rev. D 93, 074505 (2016).

[26] B. J. Choi et al. (SWME Collaboration), Phys. Rev. D 93, 014511 (2016).

[27] J. A. Bailey et al., Phys. Rev. D 89, 114504 (2014).

[28] J. A. Bailey et al. (MILC Collaboration) Phys. Rev. D 92, 034506 (2015).

[29] W. Detmold, C. Lehner, and S. Meinel, Phys. Rev. D 92 , 034503 (2015).

[30] I. Caprini, L. Lellouch, and M. Neubert, Nucl. Phys. B530, 153 (1998).

[31] I. Caprini and M. Neubert, Phys. Lett. B 380, 376 (1996).

[32] C. G. Boyd, B. Grinstein, and R. F. Lebed, Phys. Rev. D 56, 6895 (1997).

[33] C. G. Boyd, B. Grinstein, and R. F. Lebed, Nucl. Phys. B461, 493 (1996).

[34] C. G. Boyd and M. J. Savage, Phys. Rev. D 56, 303 (1997).

[35] C. G. Boyd and R. F. Lebed, Nucl. Phys. B485, 275 (1997).

[36] C. Bourrely, I. Caprini, and L. Lellouch, Phys. Rev. D 79, 013008 (2009); 82, 099902 (2010).

[37] L. Ling-Fong and H. Pagels, Phys. Rev. D 3, 2191 (1971).

[38] S. Okubo, Phys. Rev. D 3, 2807 (1971). 
[39] C. Bourrely, B. Machet, and E. de Rafael, Nucl. Phys. B189, 157 (1981).

[40] D. Bigi, P. Gambino, and S. Schacht, J. High Energy Phys. 11 (2017) 061.

[41] A. Abdesselam et al. (Belle Collaboration), arXiv: 1702.01521.

[42] F. U. Bernlochner, Z. Ligeti, M. Papucci, and D. J. Robinson, Phys. Rev. D 95, 115008 (2017); 97, 059902(E) (2018).

[43] F. U. Bernlochner, Z. Ligeti, M. Papucci, and D. J. Robinson, Phys. Rev. D 96, 091503 (2017).

[44] A. Vaquero Avils-Casco, C. DeTar, D. Du, A. El-Khadra, A. S. Kronfeld, J. Laiho, and R. S. Van de Water, EPJ Web Conf. 175, 13003 (2018).

[45] S. Hashimoto, Proceedings, 36th International Symposium on Lattice Field Theory (Lattice 2018): East Lansing, MI, USA, 2018 (Sissa, 2018), https://indico.fnal.gov/event/ 15949/session/2/contribution/325/material/slides/0.pdf.

[46] T. Kaneko et al., Proceedings, 36th International Symposium on Lattice Field Theory (Lattice 2018): East Lansing, MI, USA, 2018 (Sissa, 2018), private communication.

[47] S. Jaiswal, S. Nandi, and S. K. Patra, J. High Energy Phys. 12 (2017) 060.

[48] T. Blum et al., Phys. Rev. D 91, 074502 (2015).

[49] Z. Bai et al. (RBC and UKQCD Collaboration), Phys. Rev. Lett. 115, 212001 (2015).

[50] R. Garcia-Martin, R. Kaminski, J. R. Pelaez, J. Ruiz de Elvira, and F. J. Yndurain, Phys. Rev. D 83, 074004 (2011).

[51] G. Colangelo, J. Gasser, and H. Leutwyler, Nucl. Phys. B603, 125 (2001).

[52] https://indico.mitp.uni-mainz.de/event/48/contribution/5/ material/slides/0.pdf.

[53] S. Descotes-Genon, N. H. Fuchs, L. Girlanda, and J. Stern, Eur. Phys. J. C 24, 469 (2002).

[54] T. Wang, Proceedings, 36th International Symposium on Lattice Field Theory (Lattice 2018): East Lansing, MI, USA, 2018 (2018), https://indico.fnal.gov/event/15949/session/3/ contribution/150/material/slides/0.pdf.

[55] S. Gardner and G. Valencia, Phys. Lett. B 466, 355 (1999).

[56] V. Cirigliano, A. Pich, G. Ecker, and H. Neufeld, Phys. Rev. Lett. 91, 162001 (2003).
[57] N. H. Christ, X. Feng, G. Martinelli, and C. T. Sachrajda, Phys. Rev. D 91, 114510 (2015).

[58] N. H. Christ and Z. Bai, Proc. Sci. LATTICE2015 (2016) 342.

[59] Z. Bai, Proc. Sci. LATTICE2016 (2017) 309.

[60] A. J. Buras, D. Guadagnoli, and G. Isidori, Phys. Lett. B 688, 309 (2010).

[61] N. Christ, T. Izubuchi, C. T. Sachrajda, A. Soni, and J. $\mathrm{Yu}$ (RBC and UKQCD Collaborations), Proc. Sci. LATTICE2013 (2014) 397 [arXiv:1402.2577].

[62] A. J. Buras and D. Guadagnoli, Phys. Rev. D 78, 033005 (2008).

[63] J. Brod and M. Gorbahn, Phys. Rev. D 82, 094026 (2010).

[64] B. Chakraborty, C. T. H. Davies, B. Galloway, P. Knecht, J. Koponen, G. C. Donald, R. J. Dowdall, G. P. Lepage, and C. McNeile, Phys. Rev. D 91, 054508 (2015).

[65] J. A. Bailey, T. Bhattacharya, R. Gupta, Y.-C. Jang, W. Lee, J. Leem, S. Park, and B. Yoon (LANL-SWME Collaboration), EPJ Web Conf. 175, 13012 (2018).

[66] J. Bailey, Y.-C. Jang, W. Lee, and J. Leem (LANL-SWME Collaboration), EPJ Web Conf. 175, 14010 (2018).

[67] H. Jeong, W. Lee, J. Leem, S. Park, T. Bhattacharya, R. Gupta, and Y.-C. Jang (LANL-SWME Collaboration), Proc. Sci. LATTICE2016 (2016) 380.

[68] M. Neubert, Phys. Rep. 245, 259 (1994).

[69] K. G. Chetyrkin, J. H. Kuhn, and M. Steinhauser, Comput. Phys. Commun. 133, 43 (2000).

[70] K. Melnikov and T. v. Ritbergen, Phys. Lett. B 482, 99 (2000).

[71] F. Herren and M. Steinhauser, Comput. Phys. Commun. 224, 333 (2018).

[72] N. Gray, D. J. Broadhurst, W. Grafe, and K. Schilcher, Z. Phys. C 48, 673 (1990).

[73] P. A. Baikov, K. G. Chetyrkin, and J. H. Kuhn, Phys. Rev. Lett. 118, 082002 (2017).

[74] K. G. Chetyrkin, J. H. Kuhn, and C. Sturm, Nucl. Phys. B744, 121 (2006).

[75] P. Marquard, A. V. Smirnov, V. A. Smirnov, M. Steinhauser, and D. Wellmann, Phys. Rev. D 94, 074025 (2016).

[76] S. Bekavac, A. Grozin, D. Seidel, and M. Steinhauser, J. High Energy Phys. 10 (2007) 006. 